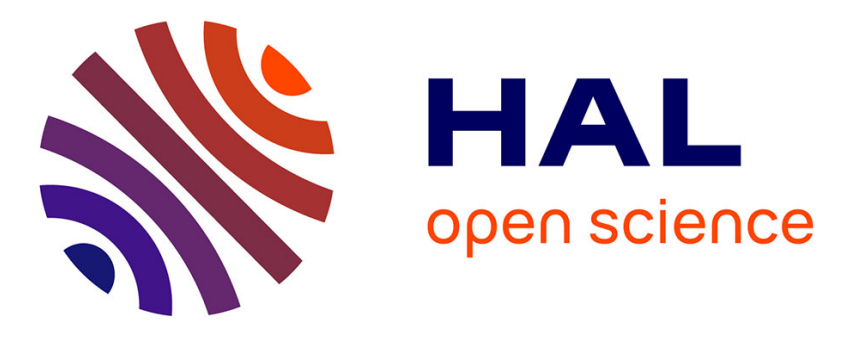

\title{
Ultrafast relaxation dynamics of highly excited hot electrons in silicon
}

Hiroshi Tanimura, Jun'Ichi Kanasaki, Katsumi Tanimura, Jelena Sjakste, Nathalie Vast

\section{- To cite this version:}

Hiroshi Tanimura, Jun'Ichi Kanasaki, Katsumi Tanimura, Jelena Sjakste, Nathalie Vast. Ultrafast relaxation dynamics of highly excited hot electrons in silicon. Physical Review B, 2019, 100 (3), 10.1103/PhysRevB.100.035201 . hal-03327955

\section{HAL Id: hal-03327955 \\ https://hal.science/hal-03327955}

Submitted on 27 Aug 2021

HAL is a multi-disciplinary open access archive for the deposit and dissemination of scientific research documents, whether they are published or not. The documents may come from teaching and research institutions in France or abroad, or from public or private research centers.
L'archive ouverte pluridisciplinaire HAL, est destinée au dépôt et à la diffusion de documents scientifiques de niveau recherche, publiés ou non, émanant des établissements d'enseignement et de recherche français ou étrangers, des laboratoires publics ou privés. 
Ultrafast relaxation dynamics of highly excited hot electrons in silicon

Hiroshi Tanimura $^{1 *}$, Jun'ichi Kanasaki ${ }^{1}$, Katsumi Tanimura ${ }^{1}$, Jelena Sjakste ${ }^{2}$, Nathalie Vast ${ }^{2}$

${ }^{1}$ The Institute of Scientific and Industrial Research, Osaka University, 8-1 Mihogaoka, Ibaraki, Osaka 567-0047, Japan

${ }^{2}$ Ecole Polytechnique, Laboratoire des Solides Irradiés, CEA-DRF-IRAMIS, CNRS UMR 7642, Institut Polytechnique de Paris, 91120 Palaiseau, France

Ultrafast relaxation dynamics of hot electrons with excess energies exceeding $1 \mathrm{eV}$ in $\mathrm{Si}$ is studied using time-resolved photoemission spectroscopy and ab initio calculations. Experimentally, the photoemission peaks from hot electrons excited in bulk electronic states along the $\Gamma-\mathrm{L}$ and $\Gamma-\mathrm{X}$ directions with excess energy $\left(\mathrm{E}_{\mathrm{ex}}\right) 1.1 \sim 3.2 \mathrm{eV}$ with respect to the conduction band minimum are identified, and the time constants that characterize the decay of transient populations are determined. The decay time, which is $30 \pm 3$ fs at $E_{e x}=3.0 \mathrm{eV}$ and increases to $115 \pm 5$ fs at $E_{e x}=1.1 \mathrm{eV}$, has the same scaling with $E_{e x}$ irrespective of the location of hot electrons in the Brillouin zone. The calculations show that the momentum scattering time due to electron-phonon coupling is shorter than $10 \mathrm{fs}$ for $\mathrm{E}_{\mathrm{ex}}$ larger than $1.5 \mathrm{eV}$, being too short to be measured. The combination of theoretical and experimental results reveals that hot electrons with high excess energy in Si are transformed into hotelectron ensembles quasi-equilibrated only in momentum space by the ultrafast momentum scattering, and that the experimentally determined time constant of population decay corresponds to the energy relaxation taking place as a whole on a time scale ten times longer than that of the momentum relaxation. The detailed methodology of the analysis of experimental data which we provide in this work, as well as our conclusions which concern the relaxation dynamics of electrons with $E_{\mathrm{ex}}$ exceeding $1 \mathrm{eV}$ in $\mathrm{Si}$, can be applied to interpret hot-carrier relaxation phenomena in a wide range of semiconducting materials.

*) Present address: Institute for Materials Research, Tohoku University, 2-1-1 Katahira, Aoba-ku, Sendai 980-8577, Japan 


\section{INTRODUCTION}

Ultrafast scattering of highly energetic carriers in semiconductors is the key process that ultimately determines functional limits and properties of nano-, micro- and optoelectronics, being a strategic research field in the past two decades $[1,2]$. For over 40 years ultrafast optical spectroscopy has been developed that complements the transport measurements by capturing scattering dynamics in the time domain $[3,4]$. Despite accumulating knowledge, a clear understanding of the physics involved in dynamic scattering processes remains elusive due to the high complexity of the problem. In particular, the understanding of ultrafast scattering processes of hot electrons in the conduction band (CB) with high excess energy $\mathrm{E}_{\mathrm{ex}}(\sim \mathrm{a}$ few $\mathrm{eV}$ above the conduction band minimum, $\mathrm{CBM})$ is still incomplete even for $\mathrm{Si}$, which is the most fundamental semiconductor and is important for the microelectronics industry.

Recent progress both in time-resolved photoemission spectroscopy $[5,6]$ and in theoretical calculations from the first principles [7-9] has made it possible to gain deeper insight into the electron relaxation dynamics. In the present paper, we study ultrafast relaxation of highly excited hot electrons with $\mathrm{E}_{\mathrm{ex}}$ ranging from $1.1 \mathrm{eV}$ to $3.2 \mathrm{eV}$ in $\mathrm{Si}$, by combining time-resolved photoemission spectroscopy and $a b$ initio theoretical study.

For electrons excited at a few $\mathrm{eV}$ above the $\mathrm{CBM}$ in $\mathrm{Si}$, the density of final electronic states available for scattering by electron-phonon (e-ph) interaction is significant, which results in the ultrafast rate of scattering from a given energy- and momentum-resolved excited state [10, 11]. In fact, a theoretical study, using Monte Carlo analysis with the empirical-pseudopotential band structure [10], demonstrated the close correlation between the scattering rate and the density of states, and evaluated the rate of the e-ph scattering of hot electrons at $2 \sim 3 \mathrm{eV}$ above the $\mathrm{CBM}$ in $\mathrm{Si}$ to be equal to 1.5$2.5 \times 10^{14} \mathrm{~s}^{-1}$, giving a scattering time of several fs. A recent $a b$ initio theoretical study has evaluated the e-ph scattering time of the energy- and momentum-resolved excited states in $\mathrm{Si}$, and an ultrafast relaxation time of 10-20 fs by e-ph interaction has been obtained, even for states excited at only 0.3 $\mathrm{eV}$ above the CBM [11]. Using the calculated relaxation time, the theory predicts that the electron 
thermalization down to the CBM occurs over $\sim 350$ fs for hot electrons generated within $1-2 \mathrm{eV}$ above the CBM [11].

In contrast to the extensive theoretical studies, experimental studies in time domain to capture the ultrafast dynamics of hot electrons with high $\mathrm{E}_{\mathrm{ex}}$ in the $\mathrm{CB}$ are still rare for $\mathrm{Si}[12,13]$. Optical techniques used to study ultrafast carrier relaxation have been restricted mainly to the measurement of transient changes in the dielectric function following excitations by laser pulses $[4,12,14]$. Despite the greater sensitivity of optical techniques, the final states of optical transitions and of e-ph scattering processes in the momentum space are inferred only indirectly. On the other hand, time-resolved twophoton photoemission spectroscopy (2PPE), including angle-resolved measurements, provides a direct spectroscopic technique to determine both the final states of optical transitions, and the energy- and momentum-resolved dynamics. In fact, important knowledge has been accumulated for ultrafast relaxation dynamics of hot electrons formed in the bulk conduction bands in several semiconductors $[15-22]$.

For $\mathrm{Si}$, Ichibayashi et al. have shown that the hot-electron population excited at $\mathrm{E}_{\mathrm{ex}}=1.1 \mathrm{eV}$ along the $\Gamma$-L direction decays with a time constant of 110 fs $[13,23-25]$, which is one order of magnitude longer than the theoretically evaluated e-ph scattering time. Furthermore, they have shown that the thermalized electron population is formed near the CBM at $660 \mathrm{fs}$ after initial hot-electron injection at $\mathrm{E}_{\mathrm{ex}}=1.2 \mathrm{eV}$. Therefore, experimentally resolved hot-electron dynamics and theoretically predicted one show significant disagreements with respect to the time scale of the hot electron relaxation. In view of the importance of $\mathrm{Si}$ to fundamental semiconductor physics and to microelectronics industry, it is of great importance to clarify the origin of the disagreement and to establish the correct interpretation of hot-electron relaxation at high excess energies.

Recently, characteristic features of hot-electron relaxation in GaAs have been found in studies based on the combination of the results obtained by time-resolved photoemission spectroscopy and by theoretical calculation from the first principles [26-27]. It has been shown that efficient momentum relaxation due to electron-phonon scattering of hot electrons excited into states with $\mathrm{E}_{\mathrm{ex}}=\sim 0.9 \mathrm{eV}$ in the $\Gamma$ valley of GaAs leads to the formation of hot-electron ensembles (HEEs) quasi-equilibrated over 
the Brillouin zone (BZ) in a very short time [26]. The energy relaxation of HEEs takes place on a timescale about ten times longer than that of the momentum relaxation [27].

Note that similar ideas have been put forward for $\mathrm{GaN}$ in [28]. The concept of HEE, established for GaAs, indicates that the transient population of hot electrons at a given state specified by the energy and momentum in the $\mathrm{CB}$ is governed by two relaxation times with different magnitudes: the momentum relaxation time $\tau_{\mathrm{M}}$ and the energy relaxation time $\tau_{\mathrm{E}}$. It is of great importance to examine the hot electron dynamics in silicon in the light of the HEE concept [29].

In the present paper, we identify photoemission peaks from hot electrons excited with high $\mathrm{E}_{\mathrm{ex}}$ in bulk electronic states along the $\Gamma$ - $\mathrm{L}$ and $\Gamma-\mathrm{X}$ directions in $\mathrm{Si}$, and capture their dynamics by timeresolved photoemission spectroscopy, to determine the population decay time at a given $\mathrm{E}_{\mathrm{ex}}$. Using state-of-the-art ab initio calculations, momentum-scattering and energy-relaxation rates are evaluated as a function of $\mathrm{E}_{\text {ex. }}$. We show that the measured population decay of hot electrons in Si at high excess energies is due to the energy relaxation of HEEs formed by ultrafast momentum scattering due to eph interaction; momentum scattering rates are shown to be too fast to be measured. Thus we emphasize that the concept of HEEs is essential in Si both to understand the relaxation processes of hot electrons in states with high $E_{e x}$, and to solve the significant discrepancy between the theoretical and experimental results reported previously.

This paper is organized as follows: Sec. II provides insights into the time-resolved two-photon photoemission spectroscopy methods. In Sec. III, the results of photoemission spectroscopy are presented first to identify the photoemission peaks from the bulk electronic states with $\mathrm{E}_{\mathrm{ex}}$ higher than the direct band gap energy $\mathrm{E}_{0}^{\prime}$ (Sec.III 1), and for determining the population decay times of the states with $\mathrm{E}_{\mathrm{ex}}$ ranging from $1.1 \mathrm{eV}$ to $3.2 \mathrm{eV}$ (III 2). In Sec. IV, we present the theoretical background for the momentum- and energy relaxation due to electron-phonon scattering, as well as technical details for numerical calculations. Finally, in Sec. V, we discuss the ultrafast relaxation processes of hot electrons in $\mathrm{Si}$, based on the experimental and theoretical results.

\section{EXPERIMENTAL METHOD}


Boron-doped p-type $\operatorname{Si}(001)$ and $\operatorname{Si}(111)$ wafers with resistivity of 4.2 and $4.8 \Omega \mathrm{cm}$ were clamped with Ta sheets to the sample holder in an ultrahigh vacuum chamber (pressure smaller than $5 \times 10^{-11}$ Torr). After appropriate thermal treatments, surface structures were characterized in situ by scanning tunneling microscopy prior to photoemission measurements. The (001) surfaces consist of well ordered double-domain (2x1)-structures with a typical surface-defect concentration of $1 \%$. The (111) surfaces showed the perfect $(7 \times 7)$ reconstructed structures, with a surface-defect concentration smaller than $0.05 \%$. A laser system, consisting of a Ti-sapphire laser oscillator, a regenerative amplifier, and a tunable optical parametric amplifier (OPA) was used to generate ultrafast laser pulses. The OPA generated 40-fs laser pulses centered at photon energies ranging from 1.7 to $2.4 \mathrm{eV}$. The second harmonics of the OPA output were used as probe-light pulses for the monochromatic 2PPE, and as pump-laser pulses in the bichromatic time-resolved photoemission measurements. Part of the amplified fundamental output at $824 \mathrm{~nm}$ or $790 \mathrm{~nm}$ was used to generate the 50 -fs third harmonic pulses for pumping the crystal and/or probing the photoemission. In the time-resolved photoemission spectroscopy, two different laser pulses with different photon energies, with a preset time delay $(\Delta t)$, were aligned co-axially and focused on sample surface at $45^{\circ}$ to the normal. Electrons emitted along the surface normal $\left( \pm 2^{\circ}\right.$, normal photoemission), were analyzed using a hemispherical analyzer with an instrumental energy resolution of $140 \mathrm{meV}$.

For $\mathrm{Si}(111)-(7 \times 7)$, electron populations in the $\mathrm{CB}$ along the $\Gamma-\mathrm{L}$ line can be monitored by measuring surface normal photoemission. The population near the CBM (close to the $\mathrm{X}$ point of the BZ) can also be detected with this geometry because of surface Umklapp processes which are numerous because the surface Brillouin zone is strongly reduced for this surface [30]. For $\mathrm{Si}(001)-(2$ $\times 1$ ), surface normal photoemission probes the states not only along the $\Gamma-X$ line but also along $2 / 3$ of the $\Sigma$-L line, due to the back folding of the electronic bands induced by the $2 \times 1$ reconstruction [31]. Therefore, the use of the two different kinds of surfaces makes it possible to characterize the relaxation pathways of highly excited electrons in $\mathrm{Si}$ in a wide momentum range, and to examine the eventual effects of the presence of surface states on the dynamics of carriers excited in bulk electronic states of Si. Regarding the sensitivity to bulk states of our experiment, the inelastic mean free path of electrons 
in solids is of a few $\AA$ for electrons with energies around $30 \mathrm{eV}$ above the Fermi level, and increases strongly when the electron energy decreases [32]. In particular, for the electron energy of $7 \mathrm{eV}$, the mean free path is $35 \AA$, which is significantly larger than the surface reconstruction depth, and thus the sensitivity to bulk properties is enhanced. Therefore, in the present bichromatic time-resolved measurements, the final state energy generated by pump and probe pulses was mostly limited to be lower than $\sim 7 \mathrm{eV}$ with respect to the valence band minimum (VBM).

\section{EXPERIMENTAL RESULTS}

A. Identification of photoemission peaks from bulk-electronic states with high excess energies in Si.

Monochromatic 2PPE using ultrashort laser pulses centered at a probe photon energy h $v_{\text {probe }}$ has been used extensively to study the energetics of unoccupied electronic states in $\mathrm{Si}[13,31,33-36]$. In this method, a first photon promotes an electron from an occupied initial state at the energy $E_{v}$ in the valence band to an intermediate state at the energy $E_{c}$ below the vacuum level $\Phi_{\text {vac }}$, and a second photon lifts the electron above $\Phi_{\text {vac }}$ to generate a photoelectron. The photoemission intensity shows a resonance enhancement when $h v_{\text {probe }}=E_{c}-E_{v}$, and the kinetic energy $E_{k i n}$ of the photoelectron indicates the energy of the intermediate state with respect to the valence band maximum (VBM). An extensive study for $\mathrm{Si}(001)-(2 \mathrm{x} 1)$ has identified several bulk-transition peaks in the photoemission spectra [31]. We use this method to identify photoemission peaks from the states above the $\Gamma_{15}^{\mathrm{c}}$ in the $\mathrm{CB}$ of $\mathrm{Si}$ with (111) surface reconstructed into the (7x7) structure.

In Fig. 1(a), we compare the $2 \mathrm{PPE}$ spectra measured by p-polarized probe light centered at $h v_{\text {probe }}=3.44 \mathrm{eV}$ and $3.70 \mathrm{eV}$ for $\mathrm{Si}(111)-(7 \times 7)$ at $293 \mathrm{~K}$. The $\mathrm{E}_{\text {kin }}$ for the spectra is given with respect to the common low-energy cutoff $E_{\text {cut }}$, which corresponds to the $\Phi_{\text {vac }}(=5.30 \mathrm{eV})$ of $\operatorname{Si}(111)-(7 \times 7)$ surfaces [30]. The upper scale of Fig. 1(a) shows the kinetic energy using $\mathrm{E}_{\mathrm{cut}}=0.65 \mathrm{eV}$. Since all states in the surface region monitored by 2PPE shift their energies in parallel, the use of $E_{\text {cut }}$ yields more reliable energies which allow to analyze the spectra in terms of electronic states of $\mathrm{Si}$, as it compensates 
possible variations of the photovoltage coming from different excitations and/or probe conditions [37]. In the figure, we see three peaks, of which two are labeled as A and B. The peaks A and B have been identified as resonance enhancements of optical transitions from the VBM to the $\Gamma_{15}^{\mathrm{c}}$ band and from $\Lambda_{3}^{\mathrm{v}}$ to $\Lambda_{1}^{\mathrm{c}}$ along the $\Gamma-\mathrm{L}$ direction, respectively [13]. When photoemission spectra are measured by s-polarized light, while the highest-energy peak A is excited, we observe that peak B is almost completely suppressed, similar to the case in Ref. 13. This is because of the polarization selection rule in the photoemission: the totally-symmetric $\left(\Lambda_{1}\right)$ final state for the normal photoemission cannot be reached by the s-polarized light from the $\Lambda_{1}^{\mathrm{c}}$ state $[38,39]$.

Figure 1 (b) shows the typical monochromatic 2PPE spectra measured by p-polarized probe light with $\mathrm{h} v_{\text {probe }}$ between 3.87 and $4.28 \mathrm{eV}$ for $\mathrm{Si}(111)-(7 \mathrm{x} 7)$ at $293 \mathrm{~K}$. For $\mathrm{h} v_{\text {probe }}>3.94 \mathrm{eV}$, the highest photoemission peak A turns into three peaks, labeled L, M, and $\mathrm{H}$. Indeed, for $\mathrm{h} v_{\text {probe }}>3.94 \mathrm{eV}$, a new peak structure (labeled L) appears on the low-energy side of peak A (peak A of panel (a) is labeled M on panel (b)), and for $h v_{\text {probe }}>4.13 \mathrm{eV}$, another peak (labeled $\mathrm{H}$ ) becomes evident on the high-energy side of peak A (peak M). Spectral decomposition analysis using the least-square fitting with three Gaussians, corresponding to peaks L, M and $\mathrm{H}$, showed that the peak $\mathrm{H}$ is present with weak intensity in the spectra for $h v_{\text {probe }}=4.00 \mathrm{eV}$ and $4.07 \mathrm{eV}$ as well. The broken curves in Fig. 1(b) show the thus resolved peak $\mathrm{H}$ in the spectra for $\mathrm{h} v_{\text {probe }}>4.00 \mathrm{eV}$. The energies of the three photoemission peaks are plotted as a function of $h v_{\text {probe }}$ (Fig. 1 (d), circular symbols). For $h v_{\text {probe }}<3.4 \mathrm{eV}$, the value of the slope in the plot is equal to 2 , and this indicates that the coherent two-photon photoemission process is the dominating one, which yields a resonant enhancement in the intensity. Contrastingly, for $h v_{\text {probe }}>3.5$ $\mathrm{eV}$, the slopes of the peaks L, M, H become smaller than 2. Blue, red and green lines in Fig. 1(d) represent the predicted theoretical kinetic energy $\left(\mathrm{E}_{\text {kin }}^{\text {th }}\right)$ for the photoemission from the states in bulk conduction bands as described below.

The intensities of the $2 \mathrm{PPE}$ spectra measured at different $h v_{\text {probe }}$ were normalized with respect to the square of the photon flux (i. e. the number of photons per unit area per unit time) to make clear the characteristics of the photon-energy dependent optical transitions. In Fig. 1(c), we show the intensities 
of the peaks B and A (peak A becomes peak $\mathrm{M}$ for $\mathrm{h} v_{\text {probe }}>3.5 \mathrm{eV}$ ) as a function of $h v_{\text {probe. }}$ The intensity of the peak B (blue circles) increases with increasing $h v_{\text {probe }}$ up to $h v_{\text {probe }}=3.4 \mathrm{eV}$, and then decreases at higher $h v_{\text {probe. }}$ On the other hand, the intensity of peak A (red circles) displays an enhancement similar to that of peak B up to $h v_{\text {probe }}=3.4 \mathrm{eV}$, then is quasi-constant up to $\mathrm{h} v_{\text {probe }}=3.9$ $\mathrm{eV}$, and then decreases. The black curve in Fig. 1 (c) shows the imaginary part $\left(\varepsilon_{2}\right)$ of the dielectric function of Si [40]. It is clear that the enhancements of the photoemission intensities of peaks A and $\mathrm{B}$ up to $\mathrm{h} v_{\text {probe }}=3.4 \mathrm{eV}$ show the same energy dependence as that of $\varepsilon_{2}$. The direct interband transitions related to critical points near the $\Gamma$ point $\left(E_{0}^{\prime}\right)$ and those along the $\Lambda$ directions $\left(E_{1}\right)$ contribute to the peak at $3.4 \mathrm{eV}$ in $\varepsilon_{2}$, while the second peak at $4.25 \mathrm{eV}$ is due to interband transitions near the $\mathrm{X}$ point in the bulk BZ [41]. In contrast to the optical spectroscopy, the normal photoemissions in the coherent 2PPE from $\mathrm{Si}(111)$ capture only transitions along the $\Gamma$ to $\mathrm{L}$ direction; interband transitions along the $\Gamma-\mathrm{X}$ cannot be monitored. In view of this momentum-resolved characteristic of the photoemission, it is reasonable to ascribe the decreasing intensities of peak A and peak B at higher energies to weaker interband transitions along the $\Gamma$ - $\mathrm{L}$ direction at the energy range where the transitions near the $\mathrm{X}$ point become dominant. Therefore, the results in Fig. 1(c) substantiate the bulk origin of the two photoemission peaks. Also, the results provide a piece of direct evidence for the previous assignments of the peaks in $\varepsilon_{2}$ made only by theoretical considerations based on the band-structure calculations.

The three-peak structure for $h v_{\text {probe }}>3.9 \mathrm{eV}$ is well resolved also in the bichromatic timeresolved photoemission measurements. Figure 2(a) shows the time-resolved photoemission spectra obtained using two different light pulses: $\mathrm{h} v_{1}=2.21 \mathrm{eV}$, while $\mathrm{h} v_{2}=4.51 \mathrm{eV}$. The time delay $\Delta \mathrm{t}$ is defined as the delay of $h v_{1}$ with respect to $h v_{2}$. Then for $\Delta t<0, h v_{1}$ pulses first excite (pump) samples and then $h v_{2}$ pulses produce photoemission (probe). On the other hand, for $\Delta t>0, h v_{2}$ pulses pump samples and photoemission is probed by h $v_{1}$ pulses. Qualitatively different features of spectroscopic evolutions are evidenced in Fig. 2(a) for the two temporal frames with positive and negative $\Delta$ t, separated by the spectrum at $\Delta \mathrm{t}=0$ shown by the red curve. When $\Delta \mathrm{t}>0$, three peaks are well resolved; peak energies are at $0.41 \mathrm{eV}, 1.07 \mathrm{eV}$ and $1.45 \mathrm{eV}$ in the spectrum at $\Delta \mathrm{t}=0$. The position of the 1.07- 
$\mathrm{eV}$ peak shows a clear low-energy shift with increasing $\Delta \mathrm{t}$ : the peak energy is $1.07 \mathrm{eV}$ at $\Delta \mathrm{t}=0$, while the energy is $0.93 \mathrm{eV}$ at $\Delta \mathrm{t}=+107 \mathrm{fs}$. As bulk electronic states provide the dominant contribution to the photoemission signal in this energy range, as shown in Fig. 1(c), we search for the possible bulk electronic states which could serve as the initial state of photoemission by $2.21-\mathrm{eV}$ light pulses.

Figure 2(b) displays a part of the Si band structure (calculated in this study) along the $\Gamma-\Lambda$ direction in the energy range relevant to the final states of optical transitions by $4.51 \mathrm{eV}$ photons. As our LDA calculation does not give accurate value of the band gap, we use the direct band gap energy $\left(\mathrm{E}_{0}{ }^{\prime}\right)$ of $3.34 \mathrm{eV}[41,42]$ (so-called "scissor shift approximation" [43, 44]) to evaluate optical transition energies in the band structure. For p-polarized 4.51-eV light, possible optical transitions from the heavy-hole $\left(\Lambda_{3}^{v}\right)$ and from light-hole $\left(\Lambda_{1}^{v}\right)$ bands are shown by red and green arrows, respectively. Figure 2(c) shows the photoemission spectra probed by s- and p-polarized 2.21-eV probe pulses at $\Delta t=13 \mathrm{fs}$, the energy being referenced to the VBM. The polarization selection rules for normal photoemission predict that the p-polarized light generates photoemission from both $\Lambda_{1}$ and $\Lambda_{3}$ states, and that s-polarized light generates photoemission from $\Lambda_{3}$ state only $[38,39]$. As the peak $\mathrm{H}$ is missing in the photoemission spectrum probed by s-polarized probe light, it is clear that the initial state of photoemission of peak $\mathrm{H}$ is the $\Lambda_{1}^{\mathrm{c}^{*}}$ band. Transitions from the $\Lambda_{3}^{\mathrm{v}}$ and $\Lambda_{1}^{\mathrm{v}}$ to $\Lambda_{1}^{\mathrm{c} \text { "band generate }}$ electronic populations at nearly the same energy. On the other hand, the photoemission peaks $\mathrm{M}$ and $\mathrm{L}$ originate mainly from the $\Lambda_{3}^{\mathrm{c}}$ state, as they are ionized by s-polarized probe light as well as by ppolarized light. The contribution from the $\Lambda_{1}^{\mathrm{c}}$ state to the peak $\mathrm{L}$ is not significant. Therefore, we conclude that three peaks labeled $\mathrm{H}, \mathrm{M}, \mathrm{L}$ are due to the states excited by optical transitions from $\Lambda_{3}^{\mathrm{v}}$ (and $\Lambda_{1}^{\mathrm{v}}$ ) to $\Lambda_{1}^{\mathrm{c} *}$, from $\Lambda_{3}^{\mathrm{v}}$ to $\Lambda_{3}^{\mathrm{c}}$, and from $\Lambda_{1}^{\mathrm{v}}$ to $\Lambda_{3}^{\mathrm{c}}$ states of the $\mathrm{CB}$, respectively.

The determination of optical transition energies based on the band structure makes it possible to evaluate theoretical kinetic energy $E_{\text {kin }}^{\text {th }}$ of photoelectrons in the monochromatic $2 \mathrm{PPE}$ measurements. For a probe photon energy $h v_{\text {probe }}$, we first identify the intermediate CB state with energy $E_{C}^{\text {th }}$ reached by the optical transition from an initial (valence-band) states with energy $E_{V}^{\text {th }}$ 
based on the relation $h v_{\text {probe }}=E_{C}^{\text {th }}-E_{V}^{\text {th. Then, }} E_{\text {kin }}^{\text {th }}$ is evaluated as $E_{\text {kin }}^{\text {th }}=\left(E_{C}^{\text {th }}+h v_{\text {probe }}\right)-\Phi_{\text {vac. }}$. The solid blue, red, and green lines in Fig. 1(d) show the calculated $E_{\text {kin }}^{\text {th }}$ as a function of $h v_{\text {probe }}$ for $2 \mathrm{PPE}$ processes associated with optical transitions from $\Lambda_{3}^{\mathrm{v}}$ to $\Lambda_{1}^{\mathrm{c} *}$, from $\Lambda_{3}^{\mathrm{v}}$ to $\Lambda_{3}^{\mathrm{c}}$, and from $\Lambda_{1}^{\mathrm{v}}$ to $\Lambda_{3}^{\mathrm{c}}$, respectively. It describes reasonably well the kinetic energy changes as a function of $h v_{\text {probe }}$. Therefore, we can conclude that the photoemission peaks detected upon excitation with $4.51-\mathrm{eV}$ photon energy for $\operatorname{Si}(111)-(7 \times 7)$ originate from the high-lying bulk electronic states $\left(\Lambda_{1}^{\mathrm{c} *}\right.$ and $\left.\quad \Lambda_{3}^{\mathrm{c}}\right)$ of Si.

For Si samples with (2x1)-reconstructed (001) surfaces, previous extensive studies have allowed to identify several bulk-transition related photoemission peaks [31]. In the photoemissions from the states above $\Gamma_{15}^{\mathrm{c}}$, the $\Delta_{5}^{\mathrm{c}}$ band plays the most important role, as the $\Delta_{2^{\prime}}^{\mathrm{c}}$ band cannot contribute to the normal photoemission $[38,39]$. We performed the bichromatic time-resolved photoemission measurements using $h v_{1}=2.21 \mathrm{eV}$ and $h v_{2}=4.82 \mathrm{eV}$ to obtain the time-resolved dynamics of hot electrons injected into the $\Delta_{5}^{\mathrm{c}}$ band.

Figure 3(a) displays a part of the theoretical band diagram of Si along the $\Gamma-\mathrm{X}$ direction. Possible transitions from the heavy hole $\left(\Delta_{3}^{\mathrm{v}}\right)$ and the light-hole $\left(\Delta_{1}^{\mathrm{v}}\right)$ bands are indicated by red and green arrows for the excitation with p-polarized 4.82-eV light. In Fig. 3(c) are shown photoemission spectra measured by s- and p-polarized $2.21-\mathrm{eV}$ light pulses at $\Delta \mathrm{t}=0 \mathrm{fs}$. The intense peak at $4.82 \mathrm{eV}$ is the photoemission from the image potential state of $\operatorname{Si}(001)-(2 x 1)$, which can be ionized only by $\mathrm{p}$ polarized probe light $[45,46]$. For s-polarized $2.21-\mathrm{eV}$ light, the intensities from the image-potential state are drastically reduced. On the other hand, the photoemission peak at $4.05 \mathrm{eV}$ can be probed with both p- and s-polarized probe light. This is consistent with the fact that symmetry considerations predict that normal photoemission from the $\Delta_{5}^{\mathrm{c}}$ bulk conduction band can be generated by both pand s-polarized light. Moreover, the peak energy of $4.05 \mathrm{eV}$ agrees reasonably well with the energetics shown in Fig. 3(a). Therefore, we conclude that the $4.05-\mathrm{eV}$ photoemission peak comes from the $\Delta_{5}^{\mathrm{c}}$ bulk conduction band of Si. In Fig. 3(b) are shown time-resolved spectra measured by s-polarized 
probe light. It is clear that the intensity is reduced when increasing $\Delta \mathrm{t}$ up to $153 \mathrm{fs}$ after excitation, and shows a shift towards the low-energy region. In Fig. 3(b), we detect one more peak at $\sim 3.1 \mathrm{eV}$, although the low-energy part of the peak could not be obtained by the low photon energy of the probe light. This low-energy peak can be attributed to the transitions between bulk states, from $\Sigma_{2}^{\mathrm{v}}$ to $\Sigma_{3}^{\mathrm{c}}$ along the $\Sigma \mathrm{L}$ line, backfolded to $\bar{\Gamma}$ by the $2 \times 1$ reconstruction of the surface as discussed in details in Ref. 31.

B. Dynamics of population decay of excited states with high excess energy

The time-resolved photoemission spectroscopy of hot electrons excited in states between $\mathrm{L}_{1}^{\mathrm{c}}$ $\left(\mathrm{E}_{\mathrm{ex}}=0.87 \pm 0.06 \mathrm{eV}\right)$ and $\Gamma_{15}^{\mathrm{c}}\left(\mathrm{E}_{\mathrm{ex}}=2.17 \mathrm{eV}\right)$ has been studied in Ref. 13. However, the temporal resolution in Ref. 13 was not sufficient to resolve the ultrafast relaxation of hot electrons in a wide excess energy range. Here we revisit ultrafast relaxation processes using higher temporal resolution in time-resolved photoemission spectroscopy.

Figures 4 (a) and 4 (c) show the normal photoemission spectra for $\operatorname{Si}(111)$ and for $\operatorname{Si}(001)$ with $\mathrm{h} v_{\text {pump }}=3.59 \mathrm{eV}$ and $\mathrm{h} v_{\text {probe }}=4.67 \mathrm{eV}$ at $\Delta \mathrm{t}=10 \mathrm{fs}$ and $450 \mathrm{fs}$. Excitation of Si with short pulses with photon energies larger than $3 \mathrm{eV}$ generates two-photon photoemission, as shown in Fig.1 (a), and the pump-pulse induced 2PPE components are always detected. As the two-photon photoemission intensities are strongly reduced for s-polarized light, s-polarized laser pulses were used as pump pulses. In order to display the spectral components originating from states transiently populated by pump pulses, we show the difference between the measured spectra at a given $\Delta \mathrm{t}$ and the spectrum measured at $\Delta \mathrm{t}=-1 \mathrm{ps}$. The spectrum measured at $\Delta \mathrm{t}=-1 \mathrm{ps}$, where no overlap between two pulses exists, is dominated by the 2PPE spectrum induced by the s-polarized 3.59-eV light. We regard the spectrum measured at $\Delta \mathrm{t}=-1 \mathrm{ps}$ as the "background" and subtract it from the measured spectra for $\Delta \mathrm{t}>-1 \mathrm{ps}$ hereafter.

In the spectra at $\Delta \mathrm{t}=10 \mathrm{fs}$, the two-peak structure consisting of peak $\mathrm{A}$ and $\mathrm{B}$ observed in monochromatic 2PPE measurements is clearly detected for both surfaces. The spectral features are 
similar with each other, although the band dispersion along the $\Gamma-\mathrm{X}$ direction is not the same as the dispersion along the $\Gamma-\mathrm{L}$ direction, as shown in Fig. 4 (b). For $\mathrm{Si}(111)$, photoemission peaks at $\mathrm{E}_{\mathrm{ex}}>$ $1.0 \mathrm{eV}$ disappear at $\Delta \mathrm{t}=450 \mathrm{fs}$, while leaving the tail of transiently occupied surface state $\mathrm{U}_{1}$ for $\mathrm{Si}(111)$ [13]. However, for $\mathrm{Si}(001)$, photoemission peaks are detected at $\mathrm{E}_{\mathrm{ex}} \simeq 0.5 \mathrm{eV}$ and $\mathrm{E}_{\mathrm{ex}}<0 \mathrm{eV}$ at $\Delta \mathrm{t}=450$ fs. The former comes from the hot electrons relaxing toward the CBM and the latter from the electrons transiently occupied surface state $\mathrm{D}_{\text {down }}[15]$.

Figure 5(a) displays a map of normal photoemission intensity as a function of $\Delta \mathrm{t}$ and $\mathrm{E}_{\mathrm{ex}}$, showing the temporal evolution of hot electrons excited along the $\Gamma$-L line. It is clear that the electron population with low $\mathrm{E}_{\mathrm{ex}}$ survives longer than that at high $\mathrm{E}_{\mathrm{ex}}$, showing that the decay time depends on $E_{e x}$ Figure 5(b) shows temporal changes in energy-resolved photoemission intensities at several $E_{e x}$ with a width of $\pm 0.07 \mathrm{eV}$. The result at $\mathrm{E}_{\mathrm{ex}}=2.4 \mathrm{eV}$ is representative of the temporal response of peak A, where coherent two-photon processes play an important role [13]. The temporal response of the intensity at $\mathrm{E}_{\mathrm{ex}}=2.4 \mathrm{eV}$ can be regarded as a measure of pump- and probe-pulse overlap. The width (full widths at half maximum) is $65 \mathrm{fs}$, which is less than $1 / 3$ of the width used in Ref. 13. Because of the possible presence of different pump-probe processes at the vicinity of $\Delta t=0$, the temporal responses shown in Fig.5(b) may not be analyzed simply by using the optical Bloch equation nor the rate equation. Therefore, we determine the decay time of energy-resolved hot-electron population based on the empirical semi-logarithmic plot of the intensity as a function of $\Delta$ t. The much improved short temporal widths of the pump and probe pulses make this method applicable to all of the states between $L_{1}^{c}$ and $\Gamma_{15}^{\mathrm{c}}$ bands, as shown in Fig.5 (b). For the states above $\Gamma_{15}^{\mathrm{c}}$ described in III.A, the same method can be applied to determine the population decay times. The population decay times thus determined are plotted as a function of $E_{e x}$ in Fig.6.

In Fig.6, the solid symbols represent the results obtained from the photoemission normal to the (111) surface of $\mathrm{Si}$, to which all states along the $\Gamma$-L direction contribute. On the other hand, the open symbols show the results obtained from the photoemission normal to the (001) surface of $\mathrm{Si}$, to which all states (except $\Delta_{2}^{\mathrm{c}}$ ) along the $\Gamma$-X direction contribute. Therefore, even at the same $\mathrm{E}_{\mathrm{ex}}$, solid and 
open symbols represent the decay times of hot-electron populations at different points of the Brillouin zone. However, the results show clearly that the population decay times are scaled uniquely in terms of $\mathrm{E}_{\mathrm{ex}}$. This is one of the most important features of the present results. Also, the population decay time shows a characteristic $E_{\text {ex }}$ dependence: it is $25 \mathrm{fs}$ at the highest $E_{\text {ex }}(=3.1 \mathrm{eV})$, stays almost constant from 2.9 to $1.9 \mathrm{eV}$, and then increases up to $120 \mathrm{fs}$ at $\mathrm{E}_{\mathrm{ex}}=1.1 \mathrm{eV}$, which is the excess energy value close to that of the minimum $\mathrm{L}_{1}^{\mathrm{c}}$ of the $\mathrm{L}$ valley of the $\mathrm{CB}$.

\section{MOMENTUM- AND ENERGY-RELAXATION RATES in Si: THEORETICAL}

\section{ANALYSIS}

\section{A. Technical details}

We describe Si within the density functional theory (DFT) in the local density approximation (LDA), with the same pseudopotentials as in our previous works $[47,48]$. The band structure obtained in our work is found in agreement with earlier calculations within the LDA approximation, and is close to the one obtained within the GW approximation, when the scissor shift operation is applied $[43,44]$. For the electronic density calculation, we used an energy cutoff value of 45 Ry to limit the size of the plane-wave basis set and a Monkhorst-Pack grid of $12 \times 12 \times 12$ points to sample the BZ. The Wannier interpolation of the electronic structure was carried out using a $8 \times 8 \times 8$ k-point grid with 10 Wannier functions and 45 DFT Bloch wave functions. The same $8 \times 8 \times 8$ grid was used as initial grid to calculate electron-phonon matrix elements within DFPT [50], which were then Wannierinterpolated in the space of maximally localized Wannier functions using the interpolation method of Ref. 49.

B. Theoretical framework

For every initial electronic state $\mid n, \mathbf{k}>$, characterized by the band index $\mathrm{n}$ and wave vector $\mathbf{k}$, we calculate the phonon emission and absorption rates $\Gamma_{n, \mathbf{k}}^{a b s}$ and $\Gamma_{n, \mathbf{k}}^{e m}$, using Fermi's Golden Rule for the phonon-mediated transition probability between one initial and one final state $\mid n, \mathbf{k}+\mathbf{q}>$ (where $\mathbf{q}$ 
is phonon wave vector), and by performing converged numerical integrals over all allowed final states $|n, \mathbf{k}+\mathbf{q}\rangle$. The effective phonon frequencies $\langle\omega\rangle_{\mathrm{nk}}^{\mathrm{emiss}}$ and $\langle\omega\rangle_{\mathrm{nk}}^{\mathrm{abs}}$ for emission and absorption (see Ref. 26 or Ref. 27) are calculated as the first momenta of the emission and absorption. In brief, the momentum scattering rate $\Gamma_{n, \boldsymbol{k}}^{M}$ is defined as

$$
\Gamma_{M}^{n, \boldsymbol{k}}=\Gamma_{n, \boldsymbol{k}}^{e m i s s}+\Gamma_{n, \boldsymbol{k}}^{a b s}
$$

On the other hand, the energy relaxation rate $\mathrm{R}_{n, \boldsymbol{k}}^{E}$ is defined as:

$$
\mathrm{R}_{\boldsymbol{E}}^{n, \boldsymbol{k}}=\langle\omega\rangle_{n, \boldsymbol{k}}^{e m i s s} \Gamma_{n, \boldsymbol{k}}^{e m i s s}-\langle\omega\rangle_{n, k}^{a b s} \Gamma_{n, \boldsymbol{k}}^{a b s}
$$

Finally, the momentum and energy scattering times $\tau_{M}$ and $\tau_{E}$ are defined, respectively, as:

$$
\tau_{M}\left(\varepsilon_{n, \mathbf{k}}\right)=\hbar / \Gamma_{n, \mathbf{k}}^{M}
$$

and

$$
\tau_{E}=\int_{\varepsilon-\Delta E}^{\varepsilon} \frac{1}{R^{E}\left(\varepsilon^{\prime}\right)} d \varepsilon^{\prime}
$$

Here, the energy-loss interval $\Delta \mathrm{E}$ depends on the experimental resolution. In this work, $\Delta \mathrm{E}=140 \mathrm{meV}$. The detailed theoretical framework and discussion of the above-defined quantities can be found in Ref. 26 or Ref. 27.

C. Results: calculated relaxation rates of hot electrons

In Figs. 7(a) and 7(b), the momentum scattering rates for the initial states along the $\Gamma-\mathrm{X}$, and $\Gamma$ $\mathrm{L}$ directions are plotted (in units of $\Gamma_{n, \boldsymbol{k}}^{M} / \hbar$ ) as a function of $\mathrm{E}_{\mathrm{ex}}$. They are essentially identical for $\mathrm{E}_{\mathrm{ex}}>2.2 \mathrm{eV}$, while the rate $\Gamma_{n, \boldsymbol{k}}^{M}$ for the initial states along the $\Gamma-\mathrm{X}$ direction is higher than that for the states along the $\Gamma$-L direction below $E_{\mathrm{ex}}=2.2 \mathrm{eV}$. However, for both kinds of initial states, the overall shape of the momentum scattering rates as a function of the excess energy is determined by the density of final electronic states, as it was already shown in previous works [11, 29]. In Fig. 7(c), the energy scattering rates $\mathrm{R}_{n, \boldsymbol{k}}^{E}$ calculated for the initial states along the $\Gamma-\mathrm{X}$, and $\Gamma$-L directions are shown; they show similar features as those of $\Gamma_{n, \boldsymbol{k}}^{M}$ in Figs. 7(a) and 7(b). Similarly to the momentum scattering rates, the overall dependence of $\mathrm{R}_{n, k}^{E}$ on $\mathrm{E}_{\mathrm{ex}}$ is mostly determined by the dependence of the density 
of final electronic on the electron energy, which is shown in Fig. 7(c). We use the results of Fig. 7 as the theoretical basis on which the characteristic features of hot electron relaxation are discussed in the next section.

\section{DISCUSSION}

Photoemission peaks from hot electrons with $\mathrm{E}_{\mathrm{ex}}$ ranging from 1.1 to $3.2 \mathrm{eV}$, generated in the bulk conduction band of $\mathrm{Si}$, have been identified unambiguously, and the temporal changes in population at a given excess energy with $\pm 70 \mathrm{meV}$ width have been determined using time-resolved photoemission measurements in section III. Decay characteristics of the populations are described by single-exponential decays with time constants dependent on $\mathrm{E}_{\mathrm{ex}}$, as shown in Figs.5 (b) and 6. Under a low density excitation, smaller than $10^{17} \mathrm{~cm}^{-3}$, it has been shown that the e-ph interaction plays the major role in the scattering process, with a minor contribution from the e-e scattering. Therefore, the e-ph interaction is the prime candidate that is responsible for the population decay of hot electrons with high excess energies. Prior to analyzing the decay characteristics of hot electrons based on the theoretical results of e-ph decay rates for energy and momentum shown in Fig.7, we discuss a few other processes that can cause possible changes in hot-electron populations, in order to evaluate their effect and/or exclude their respective contributions.

A. Changes in populations of hot electrons with high excess energies in $\mathrm{Si}$

\section{(A.1) Impact ionization}

For hot electrons with $\mathrm{E}_{\mathrm{ex}}$ larger than the band-gap energy, the impact ionization can be a mechanism leading to the decay of hot-electron populations. However, the reported rate of impact ionization in $\mathrm{Si}$ is $2 \times 10^{12}\left(\sim 1 \times 10^{11}\right) \mathrm{s}^{-1}$ at $\mathrm{E}_{\mathrm{ex}}=3.0(2.0) \mathrm{eV}$ [51]. In Ref. 52, somewhat larger values (by a factor 2) have been reported. The estimated decay time at $\mathrm{E}_{\mathrm{ex}}=3.0(2.0) \mathrm{eV}$ is $370 \pm 130(750 \pm$ 250) fs. On the other hand, the population decay time determined in the present study is 30 (40) fs at $\mathrm{E}_{\mathrm{ex}}=3.0(2.0) \mathrm{eV}$. The magnitude and $\mathrm{E}_{\mathrm{ex}}$ dependence of population decay times due to the impact 
ionization are completely different from those obtained in the present experimental results. Therefore, we can exclude the impact ionization as the mechanism leading to the ultrafast population decay revealed in the present study.

\section{(A.2) Hot-carrier diffusion}

For a quantitative analysis of the population decays of hot electrons generated under excitation with photon energies larger than $3 \mathrm{eV}$, it is crucial to take the effect of carrier diffusion into account, as the large absorption coefficient $\left(>10^{6} \mathrm{~cm}^{-1}[40]\right)$ results in a steep carrier-concentration gradient that can lead to efficient carrier transport towards bulk from the surface region. Since the present photoemission spectroscopy probes the electron dynamics within the range of typically $35 \AA$ from the surface, efficient diffusion can significantly reduce photoemission intensities. The best way to characterize the dynamical transport properties in semiconductors on a short time scale is still subject to debate. Here we use a one-dimensional diffusion-equation model for the hot-electron population $\mathrm{n}_{\mathrm{Eex}}$ at an excess energy $\mathrm{E}_{\mathrm{ex}}$ with an appropriate energy- and direction-dependent ambipolar diffusion constant $\mathrm{D}_{\mathrm{n}, \mathrm{k}}\left(\mathrm{E}_{\mathrm{ex}}\right)$ to examine the effects of hot-electron diffusion on the population decay rates. To this end, we define the $\tau_{E}^{0}$, which represents the population decay rate due to intrinsic scattering processes, and the $\tau_{E}^{a p}$, which represents the "apparent" decay rate including effects of carrier diffusion. The diffusion equation reads:

$$
\frac{\partial n_{E_{e x}}(t, z)}{\partial t}=G(t, z)-D_{n, k}\left(E_{e x}\right) \frac{\partial^{2} n_{E e x}(t, z)}{\partial z^{2}}-\frac{1}{\tau_{E}^{0}} n_{E_{e x}}(t, z)
$$

where $z$ is the distance from the surface toward bulk and $G(t, z)$ is the carrier generation rate determined by a pump-laser pulse shape and the silicon absorption coefficient $\alpha$. Recent extensive theoretical study has provided the energy- and direction-resolved mean free paths $\left(\ell_{\mathrm{n}, \mathrm{k}}\right)$ of hot electrons and holes, together with the e-ph (momentum) scattering times $\left(\tau_{\mathrm{n}, \mathrm{k}}\right)$ in a large range of energies [11]. These results enable us to estimate $\mathrm{D}_{\mathrm{n}, \mathrm{k}}$ as $\mathrm{D}_{\mathrm{n}, \mathrm{k}} \approx\left(\ell_{\mathrm{n}, \mathrm{k}}\right)^{2} / \tau_{\mathrm{n}, \mathrm{k}}$ [53]. In the case of optical excitation with photon energies less than 3.6-eV, hot electrons in the bands along the $\Gamma$ - $\mathrm{L}$ line are generated in 
$\Lambda_{1}^{\mathrm{c}}$ with $\mathrm{E}_{\mathrm{ex}}$ ranging from 1.0 to $2.0 \mathrm{eV}$, while hot holes are injected into the heavy-hole $\left(\Lambda_{3}^{\mathrm{v}}\right)$ band at energies, on average, about $0.6 \mathrm{eV}$ below the VBM. For these hot carriers, $\mathrm{D}_{\mathrm{n}, \mathrm{k}}\left(\mathrm{E}_{\mathrm{ex}}\right) \approx 4.7 \mathrm{~cm}^{2} / \mathrm{s}$ can be evaluated along the [111] direction, which is substantially smaller than the equilibrated ambipolar diffusion constant $\left(=18 \mathrm{~cm}^{2} / \mathrm{s}\right)$ [54], mainly because of the high scattering rates of electrons by phonons which are of the order of $10^{14} \mathrm{~s}^{-1}$. Similarly, $\mathrm{D}_{\mathrm{n}, \mathrm{k}}\left(\mathrm{E}_{\mathrm{ex}}\right) \approx 6 \mathrm{~cm}^{2} / \mathrm{s}$ is estimated for hot carriers with $\mathrm{E}_{\mathrm{ex}}$ ranging from 1.0 to $2.0 \mathrm{eV}$ in the bands along the $\Gamma$-X line. For hot carriers with $\mathrm{E}_{\mathrm{ex}}$ $\approx 2.5 \mathrm{eV}, \mathrm{D}_{\mathrm{n}, \mathrm{k}}\left(\mathrm{E}_{\mathrm{ex}}\right)$ along the [111] direction is $6.7 \mathrm{~cm}^{2} / \mathrm{s}$, while that along [100] direction is 14 $\mathrm{cm}^{2} / \mathrm{s}$.

Using $D_{n, k}\left(E_{e x}\right)$ estimated above, we solved the diffusion equation to obtain temporally and spatially resolved $\mathrm{n}_{\mathrm{Eex}}(\mathrm{t}, \mathrm{z})$ for a system with the depth of $1.0 \mu \mathrm{m}$ with the spatial resolution of $5 \AA$. Temporal changes of hot-electron populations within a $35 \AA \AA$ depth (from the surface) were obtained. An example of such an analysis is shown in Fig. 5(b) for the state with $\mathrm{E}_{\mathrm{ex}}=1.4 \mathrm{eV}$. The solid black curve in Fig.5 (b) displays the result calculated by putting $1 / \tau_{E}^{0}=0$ in the third term of right-hand side of Eq. (5), which amounts to taking into account the effect of hot-electron diffusion only. Although a fractional decrease in the population by about $50 \%$ is induced within the first $600 \mathrm{fs}$, the experimentally observed decay of hot electron population is substantially faster than the calculated population loss induced by diffusion only. The solid red curve is the result of Eq. (5) with $\tau_{E}^{0}=100 \mathrm{fs}$. It shows an exponential decay at $\Delta \mathrm{t}>50 \mathrm{fs}$, and the decay time $\tau_{E}^{a p}$ of $90 \mathrm{fs}$ is evaluated, which is shorter by $10 \%$ than $\tau_{E}^{0}$ used in Eq. (5). This result indicates that, in case hot-carrier diffusion yields finite effect, our empirical method of semi-logarithmic plot analysis of intensity, which determines the "apparent" decay time, may give a value which is shorter by $10 \%$ than the intrinsic $\tau_{E}^{0}$ attributed to scattering processes.

For other states with different $\mathrm{E}_{\mathrm{ex}}$ 's, a similar analysis was made, using $\mathrm{D}_{\mathrm{n}, \mathrm{k}}\left(\mathrm{E}_{\mathrm{ex}}\right), \alpha$, and $\tau_{E}^{0}$ which depend on $\mathrm{E}_{\text {ex. }}$. Although a fractional decrease in the population due to diffusion within the first $600 \mathrm{fs}$ turns out to be enhanced at $\mathrm{E}_{\mathrm{ex}}$ larger than $2.5 \mathrm{eV}$, in particular along the [100] direction, the 
decay of hot-electron population is still significantly faster than the diffusion-induced population loss. The numerical solutions of Eq. (5) always gave an exponential decay at $\Delta \mathrm{t}>50 \mathrm{fs}$ for different sets of $\mathrm{D}_{\mathrm{n}, \mathrm{k}}\left(\mathrm{E}_{\mathrm{ex}}\right), \alpha$, and $\tau_{E}^{0}$, and the decay time constants $\tau_{E}^{a p}$ were found to be shorter only by $\sim 10 \%$ than the magnitude of $\tau_{E}^{0}$ in Eq. (5). Possible effects of diffusion on population loss of hot electrons are thus limited to $\sim 10 \%$ of the intrinsic population decay rate. Therefore, we can conclude that the population decay of hot electrons with high excess energies originates mainly from intrinsic relaxation processes in the bulk conduction band of Si.

(A.3) Pump-laser induced ultrafast $\mathrm{L}_{1}-\mathrm{L}_{1}$ ' transitions

Ichibayashi et al. have first reported the ultrafast decay of hot-electron population at $\mathrm{E}_{\mathrm{ex}}=1.1 \mathrm{eV}$ in the L valley, and they ascribed the decay to the L-X intervalley scattering [13]. On the other hand, Sangalli and Marini have argued that the population loss is not the scattering from $\mathrm{L}$ to $\mathrm{X}$ valleys, but is due to the ultrafast scattering between equivalent $\mathrm{L}_{1}$ valleys which is activated by the specific polarization of the pump laser [24]. This argument is based on the theoretical result of Ref. 24, in which it was found that the decay time of the $\mathrm{L}_{1}$ population is strongly dependent on the pulse width and that excitations with the shorter pulses lead to faster decay of the $\mathrm{L}_{1}$ population. We examine here the theoretical proposal of Ref. 24 by comparing the decay kinetics of hot electrons excited by laser pulses with different temporal widths.

In Ref. 13, the pump-pulse width was 100 fs and the probe-pulse width was $180 \mathrm{fs}$, giving a cross-correlation width of $220 \mathrm{fs}$. The decay of the population at $\mathrm{E}_{\mathrm{ex}}=1.1 \mathrm{eV}$ can be determined by the semi-logarithmic plot of the photoemission intensities as a function of time delay, as in Fig.5 (b). It was $110 \pm 10 \mathrm{fs}$ in Ref. 13. In the present study, the pump-pulse width is $40 \mathrm{fs}$ and the probe-pulse width is $50 \mathrm{fs}$, giving a cross-correlation width of 65 fs. As shown in Fig.5(b), the hot-electron population at $\mathrm{E}_{\mathrm{ex}}=1.1 \mathrm{eV}$, induced by 40 -fs pump laser pulses, shows the exponential decay with the time constant of $115 \pm 5 \mathrm{fs}$, which is essentially identical to that measured for the excitation with 100 fs pump laser pulses in Ref. 13. Additionally, the decay time is not dependent on the polarization of pump-laser pulses. Therefore, we conclude that the theoretical scenario of laser-field induced ultrafast 
$\mathrm{L}_{1}-\mathrm{L}_{1}$ ' scattering by Sangalli and Marini is not relevant for the population decay of hot electrons injected into states along the $\Gamma$ - $\mathrm{L}$ direction in Si samples with (111) surfaces. Rather, the decay is induced by e-ph interactions including all $\mathrm{L}$ and $\mathrm{X}$ valleys.

(A.4) Possible effects of surface states on the hot-electron relaxation in bulk electronic states

The samples used in this study show reconstructed surface structures: (7x7)-reconstructed (111) surfaces and (2x1)-reconstructed (001) surfaces. Surface electronic structures of these reconstructed surfaces have been studied extensively. In an energy range larger than $1.0 \mathrm{eV}$ above the CBM, inverse photoemission spectroscopy has allowed to probe unoccupied surface states: $U_{2}$ for the (111)- $(7 \times 7)$ surface [55] and the dimer-bond state for the $(001)-(2 \times 1)$ surface $[36,55]$. The high-energy surface levels are degenerate with strongly dispersing bulk conduction bands, and form surface-resonance states with strong electronic interaction between the surface levels and bulk states, so that Fano's resonances can often be observed in spectroscopic studies [46]. The lifetime of a surface resonance state is typically as short as $10 \mathrm{fs}$ [46], and the dynamics may be difficult to capture directly by the present temporal width of 40 (50) fs of pump (probe) pulses. In fact, we could not detect any signatures of surface resonance peaks in our study of Si surfaces.

One important feature of the surface resonance states is that the interactions with bulk states are limited to the near-surface region, because the wavefunctions of the surface levels are spatially extended only at the surface region, typically within a depth of a few $\AA$ [56]. As the present photoemission spectroscopy probes hot electrons in the depth of $35 \AA$ from the surface, the fraction of bulk states affected by the surface resonances is typically $1 / 10$. Thus, the main characteristics of the relaxation process of bulk electronic states may not be affected by the surface states. In fact, a previous time-resolved photoemission spectroscopy study of $\operatorname{InP}(100)$ surfaces has demonstrated clearly that the energy loss rate of the photoexcited electron distribution is mostly determined by inelastic electronphonon scattering between bulk states, without any detectable changes attributed to the surface states [19]. As shown in Fig.6, the population decay times in states with $\mathrm{E}_{\mathrm{ex}}>1.5 \mathrm{eV}$ measured for samples with (111)-(7x7) and (001)-(2x1) surfaces are identical and show the same dependence on $\mathrm{E}_{\mathrm{ex}}$. For 
both surfaces, no singularities are detected in the energy region where surface specific states are located. This result supports our conclusion that the surface-specific states including surface defects do not give any serious effects on the population decay times of excited electrons in the bulk conduction band.

B. The hot-electron energy relaxation by the electron-phonon interaction in $\mathrm{Si}$

Based on the discussions presented in section $\mathrm{V}$ A, we conclude that the population decays of highly excited electrons determined in this study are due to the relaxation processes in the bulk conduction band of Si governed by e-ph interactions.

Theoretically, we have evaluated the momentum scattering time $\tau_{M}$ for initial states along $\Gamma$ $\mathrm{L}$ and $\Gamma$-X directions with $\mathrm{E}_{\mathrm{ex}}$ ranging from 1 to $3 \mathrm{eV}$. The magnitude of $\tau_{M}$ changes from $3 \mathrm{fs}$ (at $\mathrm{E}_{\mathrm{ex}}=2.85 \mathrm{eV}$ ) to $15 \mathrm{fs}$ (at $\mathrm{E}_{\mathrm{ex}}=1.05 \mathrm{eV}$ ). These results are consistent with the ones reported in Ref. 11 . The ultrafast momentum scattering processes transfer optically generated hot electrons to other points of the BZ, and induce redistribution of hot-electron populations in the momentum space. The present theoretical results show that at $\mathrm{E}_{\mathrm{ex}}=1.10 \mathrm{eV}$, which is the energy close to the minimum of the $\mathrm{L}$ valley, the momentum scattering time $\tau_{M}^{X}$ of hot electrons with the initial state in X valley is $12 \mathrm{fs}$, while the time $\tau_{M}^{L}$ for electrons in L valley is 15 fs. As the time $\tau_{\mathrm{qe}}$ required for quasi-equilibration of hot electrons in the momentum space is given by $1 / \tau_{q e}=1 / \tau_{M}^{X}+1 / \tau_{M}^{L}$ [57], quasi-equilibrium is established within $6.7 \mathrm{fs}$. In the region of higher $\mathrm{E}_{\mathrm{ex}}$, the magnitude of $\tau_{\mathrm{qe}}$ becomes even shorter: $\tau_{\mathrm{qe}}=1.4 \mathrm{fs}$ is estimated for $\mathrm{E}_{\mathrm{ex}}=2.9 \mathrm{eV}$. As the temporal widths of the pump and probe pulses in the present experiment are 40 and $50 \mathrm{fs}$, it is not possible to resolve directly such ultrafast processes with time constants smaller than $10 \mathrm{fs}$. Therefore, it is reasonable to conclude that the present time-resolved measurements probe changes in hot-electron populations after quasi-equilibrated distributions of hot electrons in momentum space are formed.

Experimentally, the population decay times of hot electrons have been determined as a function of $E_{\mathrm{ex}}$. As seen in Fig.6, the time constant of $25 \mathrm{fs}$ at $\mathrm{E}_{\mathrm{ex}}=3.0 \mathrm{eV}$ stays almost constant in the range of 
$E_{e x}$ from 2 to $3 \mathrm{eV}$, and then becomes longer below $E_{e x}=2 \mathrm{eV}$. The decay of the population $\mathrm{n}_{\text {Eex }}$, from a state with a certain excess energy $E_{e x}$, represents the energy relaxation, and is characterized by the time constant of the population decay. The shift of photoemission peaks to low-energy side observed in Figs. 2, 3 and 5 indicates clearly that the decay process is associated with the energy relaxation of hot electrons. Based on this characteristic feature, and taking into account the theoretically predicted ultrafast momentum scattering rates for these states, we conclude that the hot electrons in $\mathrm{Si}$ are quasi-equilibrated in the momentum space, forming the hot electron ensembles, as in GaAs $[26,27]$. As shown in Fig.6, the experimentally determined time constant which characterizes the population decay at a given $\mathrm{E}_{\mathrm{ex}}$ is common for states located at different points in the momentum space. This feature reveals that hot electrons at a given $\mathrm{E}_{\mathrm{ex}}$ in the $\mathrm{BZ}$ have the same population-decay characteristics, which implies quasi-equilibration of hot electrons in the momentum space. Also, the fact that normal photoemission spectra representing hot electron populations along the $\Gamma$ - $\mathrm{L}$ direction and $\Gamma$-X directions at $\Delta \mathrm{t}=20 \mathrm{fs}$ show very similar spectroscopic features (Fig.4), is totally consistent with the consequences of forming the HEEs.

For HEEs, the population decay time at a given $\mathrm{E}_{\mathrm{ex}}$ can be described in terms of energy-relaxation time defined in Eq.(5). For later convenience, we abbreviate the energy relaxation rates for the states prepared along the $\Gamma$-X $(\Gamma-\mathrm{L})$ line as $R_{E}^{X}\left(R_{E}^{L}\right)$. As shown in Fig. 7(c), the energy relaxation rates show characteristic dependence on $\mathrm{E}_{\mathrm{ex}} ; R_{E}^{X}$ and $R_{E}^{L}$ are essentially identical above $\mathrm{E}_{\mathrm{ex}}>2.2 \mathrm{eV}$, while the rate $R_{E}^{X}$ is larger by $\sim 40 \%$ than $R_{E}^{L}$ below $\mathrm{E}_{\mathrm{ex}}=2.2 \mathrm{eV}$. As the energy relaxation rate is determined by the effective phonon frequencies and electron-phonon scattering rates for emission and absorption, the characteristic features of $R_{E}^{n, k}$ reflect the $\mathrm{E}_{\mathrm{ex}}$-dependent changes in these quantities. In order to elucidate the physical origin of the difference between $R_{E}^{X}$ and $R_{E}^{L}$ below $\mathrm{E}_{\mathrm{ex}}=2.2 \mathrm{eV}$, we analyze "the final region-specific momentum scattering rates" below.

The concept of "valley" is well defined only near a local minimum of the CB. In order to get insight into the relative roles of different scattering channels in the electron-phonon scattering, we introduce the local regions ("L-valley region" and "X-valley region") close to the $\mathrm{L}$ and $\mathrm{X}$ valley minima in the BZ, based on the comparison of the distances from a given $\boldsymbol{k}$ '-point (which defines the 
final electronic state: $\mathbf{k}^{\prime}=\mathbf{k}+\mathbf{q}$ ) to L and X valley minima of the lowest conduction band [29]. We apply this separation only to scatterings form initial states with $\mathrm{E}_{\mathrm{ex}}$ below $1.9 \mathrm{eV}$.

For hot electrons with a given $\mathrm{E}_{\mathrm{ex}}$ and the initial state e.g. in the L-valley region, three different scattering processes can contribute to the energy relaxation: the relaxation within the same L-valley region, the transitions to the equivalent L-valley regions (L-L scattering), and the transitions to the Xvalley regions (L-X scattering). The relaxation in the same L-valley region is dominated by acoustic phonons in Si. Because of the small phase space available for the scattering and low frequencies of emitted phonons, the contribution of the intravalley scattering to the energy loss rate is very small. In contrast, the L-L and L-X scatterings provide efficient channels of energy relaxation. By restricting the range of integration over the final electronic states to $\mathrm{L}$ or $\mathrm{X}$ regions in the $\mathrm{BZ}$, we evaluated separately the final region-specific momentum scattering rates for L-L, L-X, X-L, and X-X scattering processes: $\Gamma_{M}^{L L}, \Gamma_{M}^{L X}, \Gamma_{M}^{X L}$, and $\Gamma_{M}^{X X}$. The results are shown in Figs. 7(a) and 7(b). The higher rate of the L-X scattering than that of the L-L scattering comes from the larger density of states at the X-valley region which makes the $\Gamma_{\mathbf{M}}^{n, k}$ larger. Similarly, the X-X scattering rate is higher than the X-L scattering rate. The effective emitted phonon frequencies in the X-X and X-L scatterings are found to be of $45 \pm 1.0 \mathrm{meV}$, while those for the $\mathrm{L}-\mathrm{X}$ scatterings are equal to $36 \pm 3 \mathrm{meV}$ in the $\mathrm{E}_{\text {ex }}$ range from 1.05 to $2.05 \mathrm{eV}$. The $\mathrm{X}-\mathrm{X}$ scattering is found to be the most efficient channel for the energy relaxation of hot electrons, because of the large density of final electronic states, large effective frequency of emitted phonons and the average electron-phonon matrix elements which are found to be somewhat larger for X-X scatterings as compared to L-X ones. This results in the higher $R_{E}^{X}$ than $R_{E}^{L}$ at $\mathrm{E}_{\mathrm{ex}}$ below $2.0 \mathrm{eV}$, as shown in Fig.7(c).

Although theoretically evaluated $R_{E}^{X}$ is higher than $R_{E}^{L}$ for $\mathrm{E}_{\mathrm{ex}}<2.2 \mathrm{eV}$, the experimental results of the population decay time of hot electrons for the initial states along the $\Gamma$ - $L$ and $\Gamma-\mathrm{X}$ directions are identical as shown in Fig.6. This is a clear indication that the hot-electron populations at $\mathrm{X}$ and $\mathrm{L}$ valleys are not independent; they are quasi-equilibrated by the ultrafast momentum scattering. Based on the detailed balance relation [57], we can take the statistical average of the $R_{E}^{X}$ and $R_{E}^{L}$ for the HEE; 


$$
R_{E}\left(E_{e x}\right)=\frac{\Gamma_{M}^{X L}}{\Gamma_{M}^{L X}+\Gamma_{M}^{X L}} R_{E}^{L}+\frac{\Gamma_{M}^{L X}}{\Gamma_{M}^{L X}+\Gamma_{M}^{X L}} R_{E}^{X}
$$

where $\Gamma_{M}^{L X}, \Gamma_{M}^{X L}$ are the final region-specific momentum scattering rates for L-X and X-L scattering processes, defined above.

Using the theoretical results presented in Fig.7, we have evaluated $R_{E}\left(E_{e x}\right)$ for $E_{e x}<1.9 \mathrm{eV}$. For $\mathrm{E}_{\mathrm{ex}}>1.9 \mathrm{eV}$, we used the $\Gamma_{M}^{L X}$ and $\Gamma_{M}^{X L}$ values obtained at $\mathrm{E}_{\mathrm{ex}}=1.9 \mathrm{eV}$. Note that for $\mathrm{E}_{\mathrm{ex}}>2.2 \mathrm{eV}$, $R_{E}^{X}$ and $R_{E}^{L} \quad$ are almost the same, and that the method of averaging does not play any role. The solid curve in Fig. 6 is the calculated energy relaxation time $\tau_{E}$ based on the thus determined $R_{E}\left(E_{e x}\right)$, and $\Delta \mathrm{E}=0.14 \mathrm{eV}$ was used in Eq. (5). It is clear that the theoretical $\tau_{\mathrm{E}}$ shows essentially the same $E_{\text {ex}}$-dependence as the experimental population decay time, although the absolute values of the theoretical $\tau_{\mathrm{E}}$ are smaller than the experimental ones. This shows clearly that the main features of the excess-energy dependence of the experimental population decay time are determined by the density of final electronic states available for electron-phonon scattering. One has to note that this is the first time, to the best of our knowledge, when the dominant role of the electronic density of states in the magnitude of the electron-phonon relaxation (well established by the theory) is evidenced experimentally over such a large range of excess energies.

Our theoretical results show that the momentum scattering time from the $\mathrm{L}$ to $\mathrm{X}$ valleys at $\mathrm{E}_{\mathrm{ex}}=1.1 \mathrm{eV}$ is $20 \mathrm{fs}$, which is far shorter than the experimentally determined population decay time $(115 \pm 5 \mathrm{fs})$ at $\mathrm{E}_{\mathrm{ex}}=1.1 \mathrm{eV}$ at the $\mathrm{L}$ valley. As described above, the momentum and energy relaxation take place at drastically different timescales. Under the presence of ultrafast momentum scatterings among all states, a population loss for a given initial state $\mid n, \mathbf{k}>$ due to the scattering to other states is compensated partly by the back scatterings to the state $\mid n, \mathbf{k}>$, which results in a suppression of the net population loss. As phonon emission largely dominates over phonon absorption, such effects by ultrafast momentum scattering will continue until the energy of the HEE is lowered to such an extent that the state $\mid n, \mathbf{k}>$ is no longer filled by scatterings from other states via the momentum scattering. 
Therefore, for hot electrons far from the conduction band minimum, the decay of hot-electron population at a given valley should be re-interpreted with the concept of hot-electron ensemble.

Finally, we briefly discuss possible reasons of a difference between experimental and theoretical results for the energy relaxation rate. As seen in Fig.6, the theoretical values of the energy relaxation times are smaller than the population decay times determined experimentally. When we take the effects of the hot-electron diffusion into account, the difference becomes even larger by another $10 \%$ of the intrinsic population decay rate. In the present theoretical studies, electron-phonon interaction was evaluated at the low-excitation density limit without introducing any effects of screening by laser-induced electron-hole pairs. Previous theoretical and experimental studies have shown that the screening of e-ph interaction becomes significant for values of the excitation density above $\sim 1 \times 10^{21} \mathrm{~cm}^{-3}$ [58-60]. In the present experiments, however, the excitation densities by pumplaser pulses are not higher than $1 \times 10^{19} \mathrm{~cm}^{-3}$. Therefore, any effects of screening on e-ph interaction can be neglected.

We presume that the mismatch between the theoretical results and experimental ones may be due to the cascade relaxation processes of hot electrons in the experiments; hot electrons at higher energy region flow into the lower-energy groups in the relaxation process. When the population flows from high-lying states into the hot-electron group with a given $E_{e x}$, the net rate of population decay is reduced, resulting in a longer population decay time. In our previous study for GaAs [26, 27], the population decay time of hot electrons and the theoretical energy-relaxation time show excellent agreement. In the case of GaAs, because of the direct-gap character of the material, it was possible to generate selectively hot electrons with different excess energies by tuning pump-photon energy. The population decay times of the hot electrons with the highest excess energies (to which population flows from high-lying states were negligible) could be analyzed. On the other hand, in the present study for $\mathrm{Si}$, such a fine tuning to generate selectively hot-electron packets with different $\mathrm{E}_{\mathrm{ex}}$ was not possible, and hot electrons with a wide $\mathrm{E}_{\mathrm{ex}}$ range are generated as shown in Fig.4. Therefore, population flows from high-lying states into the hot-electron group with a given $\mathrm{E}_{\mathrm{ex}}$ may not be neglected. The full description of this population-flow effect requires a realistic description of the energy- and time- 
dependent populations of excited electrons using more sophisticated theoretical approaches. This is beyond of the scope of the present work, and will be a future issue.

\section{SUMMARY}

We have studied the ultrafast relaxation dynamics of hot electrons with high excess energies in $\mathrm{Si}$, using time-resolved photoemission spectroscopy and ab initio calculations. Monochromatic 2PPE spectroscopy has allowed us to identify the photoemission peaks from hot electrons excited in bulk electronic states along the $\Gamma$-L and $\Gamma$-X directions above the direct band gap, and the population-decay times have been determined as a function of $\mathrm{E}_{\text {ex }}$ ranging from 1.1 to $3.2 \mathrm{eV}$. The decay time scales uniquely with $E_{e x}$, irrespective of the location of the initial state in the Brillouin zone, and shows an $E_{e x}$-dependent change: the decay time of $30 \pm 3$ fs at $E_{\mathrm{ex}}=3.0 \mathrm{eV}$ increases with decreasing $E_{\mathrm{ex}}$ to 110 fs at $E_{e x}=1.1 \mathrm{eV}$. We have argued that the population decay is governed by the e-ph interaction by excluding the possible effects of the impact ionization, of the laser-field induced $\mathrm{L}_{1}-\mathrm{L}_{1}$ ' scattering, and of the presence of surface defects. However, we have evaluated a finite contribution of efficient hotelectron diffusion which shortens the decay times typically by $10 \%$. Based on the extensive theoretical results of momentum and energy relaxation times calculated as a function of $E_{\text {ex }}$, we have concluded that hot electrons with high excess energy in Si are transformed into hot-electron ensembles quasiequilibrated in momentum space by ultrafast momentum scattering, and that the energy relaxation takes place as a whole on a longer time scale than the momentum relaxation, with rates dependent only on the excess energy. By comparing experimental and theoretical results for the energy relaxation times, we have provided an experimental proof of the dominant role played by the density of final electronic states in the energy relaxation due to the electron-phonon scattering, over a wide region of excess energies of hot electrons.

Acknowledgements 
This work was supported by the Japan Society for the Promotion of Science (JSPS) KAKENHI Grant No. 24000006. This work was also supported by LABEX PALM (ANR-10-LABX-0039PALM, Project Femtonic). Computational results have been obtained with the QUANTUM ESPRESSO [61] and WANNIER90 [62] packages. Computer time has been granted by GENCI CINES (Project 2210) and by Ecole Polytechnique through the LLR-LSI project. 
References

1) C. Delerue and M. Lannoo, Nanostructures: Theory and Modeling, Nanoscience and Technology (Springer Verlag, Berlin, 2004).

2) A. Polman and H. A. Atwater, Nat. Mater. 11, 174 (2012).

3) F. Rossi and T. Kuhn, Rev. Mod. Phys. 74, 895 (2002).

4) J. Shah, Ultrafast Spectroscopy of semiconductors and Semiconductor Nanostructures, 2nd ed. (Springer, Berlin, 1999).

5) H. Petek and S. Ogawa, Prog. Surf. Sci. 56, 239 (1997).

6) M. Bauer, A. Marienfeld, and M. Aeschlimann, Prog. Surf. Sci. 90, 319 (2015).

7) N. Vast, J. Sjakste, G. Kané, and V. Trinité, in Simulation of Transport in Nanodevices vol. 1, (London, Wiley, 2016).

8) M. Bernardi, Eur. Phys. J. B 89, 15 (2016).

9) F. Giustino, Rev. Mod. Phys. 89, 015003 (2017), and refererences herein.

10) M. V. Fischetti and S. E. Laux, Phys. Rev. B 38, 9721 (1988).

11) M. Bernardi, D. Vigil-Fowler, J. Lischner, J. B. Neaton, and S. G. Louie, Phys. Rev. Lett. 112, 257402 (2014).

12) F. Doany and D. Grischkowsky, Appl. Phys. Lett. 52, 36 (1988).

13) T. Ichibayashi, S. Tanaka, J. Kanasaki, K. Tanimura, and T. Fauster, Phys. Rev. B 84, 235210 (2011).

14) S. K. Cushing, M. Zürch, P. M. Kraus, L. M. Carneiro, A. Lee, H-T. Chang, C. J. Kaplan, and .S R. Leone, Struct. Dyn. 5, 054302 (2018).

15) T. Ichibayashi and K. Tanimura, Phys. Rev. Lett. 102, 087403 (2009).

16) L. Töben, L. Gundlach, R. Ernstorfer, R. Eichberger, T. Hannappel, F. Willig, A. Zeiser, J. Förstner, A. Knorr, P. H. Hahn, and W. G. Schmidt, Phys. Rev. Lett. 94, 067601 (2005).

17) J. Kanasaki, H. Tanimura, and K. Tanimura, Phys. Rev. Lett. 113, 237401 (2014).

18) H. Tanimura, J. Kanasaki, and K. Tanimura, Phys. Rev. B 91, 045201 (2015). 
19) P. Sippel, J. M. Szarko, T. Hannappel, and R. Eichberger, Phys. Rev. B 91, 115312 (2015).

20) W. A. Tisdale, M. Muntwiler, D. J. Norris, E. S. Aydil, and X.-Y. Zhu, J. Phys. Chem. C 112, 14682 (2008).

21) J.-C. Deinert, D. Wegkamp, M. Meyer, C. Richter, M. Wolf, and J. Stähler, Phys. Rev. Lett. 113, 057602 (2014).

22) A. Argondizzo, X. Cui, C. Wang, H. Sun, H. Shang, J. Zhao, and H. Petek, Phys. Rev. B 91, 155429 (2015).

23) In Ref. 13, the authors observed the population-decay time of $110 \pm 10 \mathrm{fs}$ at $E_{\mathrm{ex}}=1.1 \mathrm{eV}$. Then, they have used the diffusion equation model to evaluate the contributions attributed to the carrierdiffusion effect and to the the L-X intervalley transition. Based on this analysis, they concluded that the L-X intervalley transition time was $180 \mathrm{fs}$. Here we cite the experimentally observed value of $110 \mathrm{fs}$

24) D. Sangalli and A. Marini, Euro Phys. Lett. 110, 47004 (2015).

25) The-population decay time of 110 fs, measured in Ref. 13 (see also Ref. 23) has been interpreted in Ref. 24 with a different scenario in which pump-laser induced L-L inter-valley transitions have been proposed. The applicability of the scenario proposed in Ref. 24 is discussed critically in Sec. V of the present work.

26) H. Tanimura, J. Kanasaki, K. Tanimura, J. Sjakste, N. Vast, M. Calandra, and F. Mauri, Phys. Rev. B 93, 161203(R) (2016).

27) J. Sjakste, N. Vast, G. Barbarino, M. Calandra, F. Mauri, J. Kanasaki, H. Tanimura, and K. Tanimura, Phys. Rev. B 97, 064302 (2018).

28) V. P. Zhukov, V. G. Tyuterev, E. V. Chulkov, and P. M. Echenique, J. App. Phys. 120, 085708 (2016).

29) J. Sjakste, K. Tanimura, G. Barbarino, L. Perfetti, and N. Vast, J. Phys.: Condens. Matter, 30, 353001 (2018).

30) T. Ichibayashi and K. Tanimura, Phys. Rev. B 75, 235327 (2007). 
31) C. Kentsch, M. Kutschera, M. Weinelt, T. Fauster, and M.Rohlfing, Phys. Rev. B 65, 035323 (2001).

32) K. Oura, V. G. Lifshits, A. A. Saranin, A. V. Zotov, and M. Katayama, Surface Science: An Introduction (Springer, Berlin, 2003).

33) K. Shudo and T. Munakata, Phys. Rev. B 63, 125324 (2001).

34) K. Shudo and T. Munakata, Phys. Rev. B 65, 075302 (2002).

35) W. Schattke, E. E. Krasovskii, R. D. Muiño, and P. M. Echenique, Phys. Rev. B 78, $155314(2008)$.

36) T. Fauster, S. Tanaka, and K. Tanimura, Phys. Rev. B 84, 235444 (2011).

37) M. Weinelt, M. Kutschera, R. Schmidt, C. Orth, T. Fauster, M. Rohlfing, Appl. Phys. A 80, 995 (2005).

38) W. Eberhardt and F. J. Himpsel, Phys. Rev. B 21, 5572 (1980).

39) D. W. Niles, D. Rioux and H. Hochst, Phys. Rev. B 46, 12547 (1992).

40) D. E. Aspnes and A. A. Studna, Phys. Rev. B 27, 985 (1983).

41) P. Lautenschlager, M. Garriga, L. Vina, and M. Cardona, Phys. Rev. B 36, 4821 (1987).

42) O. Madelung, Semiconductors—Basic Data, 2nd ed. (Springer, New York, 1996).

43) K. A. Johnson, N. W. Ashcroft, Phys. Rev. B 58, 15548 (1998).

44) M. Schlüter, and L.J. Sham "Density-Functional Theory of the Band Gap", Advances in Quantum Chemistry, Vol. 21, 97-112 (1990).

45) M. Kutschera, M. Weinelt, M. Rohlfing, and T. Fauster, Appl. Phys. A 88, 519 (2007).

46) C. Eickhoff, M. Teichmann, and M. Weinelt, Phys. Rev. Lett. 107, 176804 (2011).

47) V. G. Tyuterev, J. Sjakste, N. Vast, Phys. Rev. B 81, 245212 (2010).

48) Z. Wang, S. Wang, S. Obukhov, N. Vast, J. Sjakste, V. Tyuterev, and N. Mingo, Phys. Rev. B 83, 205208 (2011). 
49) M. Calandra, G. Profeta, and F.Mauri, Phys. Rev. B 82, 165111 (2010).

50) S. Baroni, S. de Gironcoli, A. D. Corso, and P. Giannozzi, Rev. Mod. Phys. 73, 515 (2001).

51) T. Kotani and M. van Schilfgaarde, Phys. Rev. B 81, 125201 (2010).

52) N. Sano and A. Yoshii, J. Appl. Phys. 77, 2020 (1995).

53) C. Kittel, Thermal Physics, (John Wiley \& Sons, New York, 1969).

54) J. F. Young and H. M. van Driel, Phys. Rev. B 26, 2147 (1982).

55) F.J. Himpsel, Surf. Sci. Rept. 12, 1 (1990).

56) W. G. Schmidt, N. Esser, A. M. Frisch, P. Vogt, J. Bernholc, F. Bechstedt, M. Zorn, T. Hannappel, S. Visbeck, F. Willig, and W. Richter, Phys. Rev. B 61, R16335 (2000).

57) C. J. Stanton and D. W. Bailey, Phys. Rev. B 45, 8369 (1992).

58) E. J. Yoffa, Phys. Rev. B 21, 2415 (1980), and ibid. B 23, 1909 (1981).

59) T. Sjodin, H. Petek, and H. -L. Dai, Phys. Rev. Lett. 815664 (1998).

60) M. Harb, R. Ernstorfer, T. Dartigalongue, C. T. Hebeisen, R. E. Jordan, and R. J. Dwayne Miller, J. Phys. Chem. B 110, 25308 (2006).

61) P. Giannozzi, O. Andreussi, T. Brumme, O. Bunau, M. B. Nardelli, M. Calandra, R. Car, C. Cavazzoni, D. Ceresoli, M. Cococcioni et al., J. Phys.: Condens. Matter, 29, 465901 (2017).

62) N. Marzari, A. A. Mostofi, J. R. Yates, I. Souza, and D. Vanderbilt, Rev. Mod. Phys. 84, 1419 (2012). 
Figures and Figure Captions
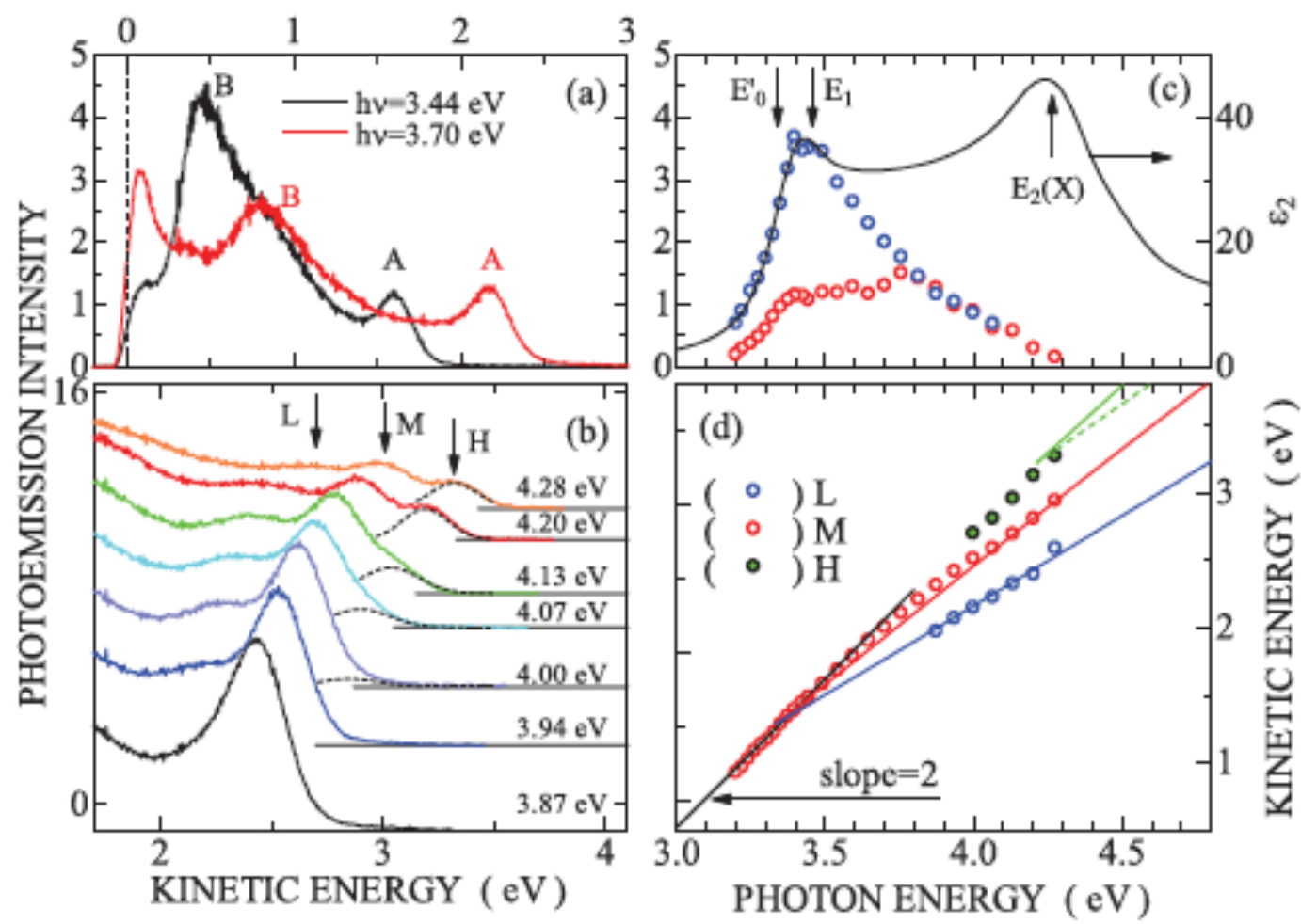

Fig.1. (a) Two-photon photoemission spectra from $\operatorname{Si}(111)-(7 x 7)$ measured for p-polarized probe pulses at $h v_{\text {probe }}=3.44 \mathrm{eV}$ and $3.70 \mathrm{eV}$ at $293 \mathrm{~K}$. For the three peaks in the both spectra, two peaks at higher energies are labeled as A and B. (b) Two-photon photoemission spectra from $\mathrm{Si}(111)-(7 \times 7)$ measured for p-polarized probe pulses at $\mathrm{h} v_{\text {probe }}>3.8 \mathrm{eV}$. The peak A splits into three peaks labeled as $\mathrm{L}, \mathrm{M}$, and $\mathrm{H}$. The spectra for $\mathrm{h} v_{\text {probe }}>3.94 \mathrm{eV}$ are offset for clarity with base lines shown by black lines. (c) The intensities of peak A (red) and B (blue) as a function of probe-photon energy. The black curve shows the imaginary part of dielectric function of Si. (d) The photoelectron kinetic energies at the peak of peaks A, L, M, and $\mathrm{H}$ as a function of probe-photon energy. The solid (broken) green, red and blue curves are the theoretical kinetic energies of photoelectrons associated with the transitions from $\Lambda_{3}^{\mathrm{v}}\left(\Lambda_{1}^{\mathrm{v}}\right)$ to $\Lambda_{1}^{\mathrm{c} *}$, from $\Lambda_{3}^{\mathrm{v}}$ to $\Lambda_{3}^{\mathrm{c}}$, and from $\Lambda_{1}^{\mathrm{v}}$ to $\Lambda_{3}^{\mathrm{c}}$, based on the band structure of Si (see the text). 


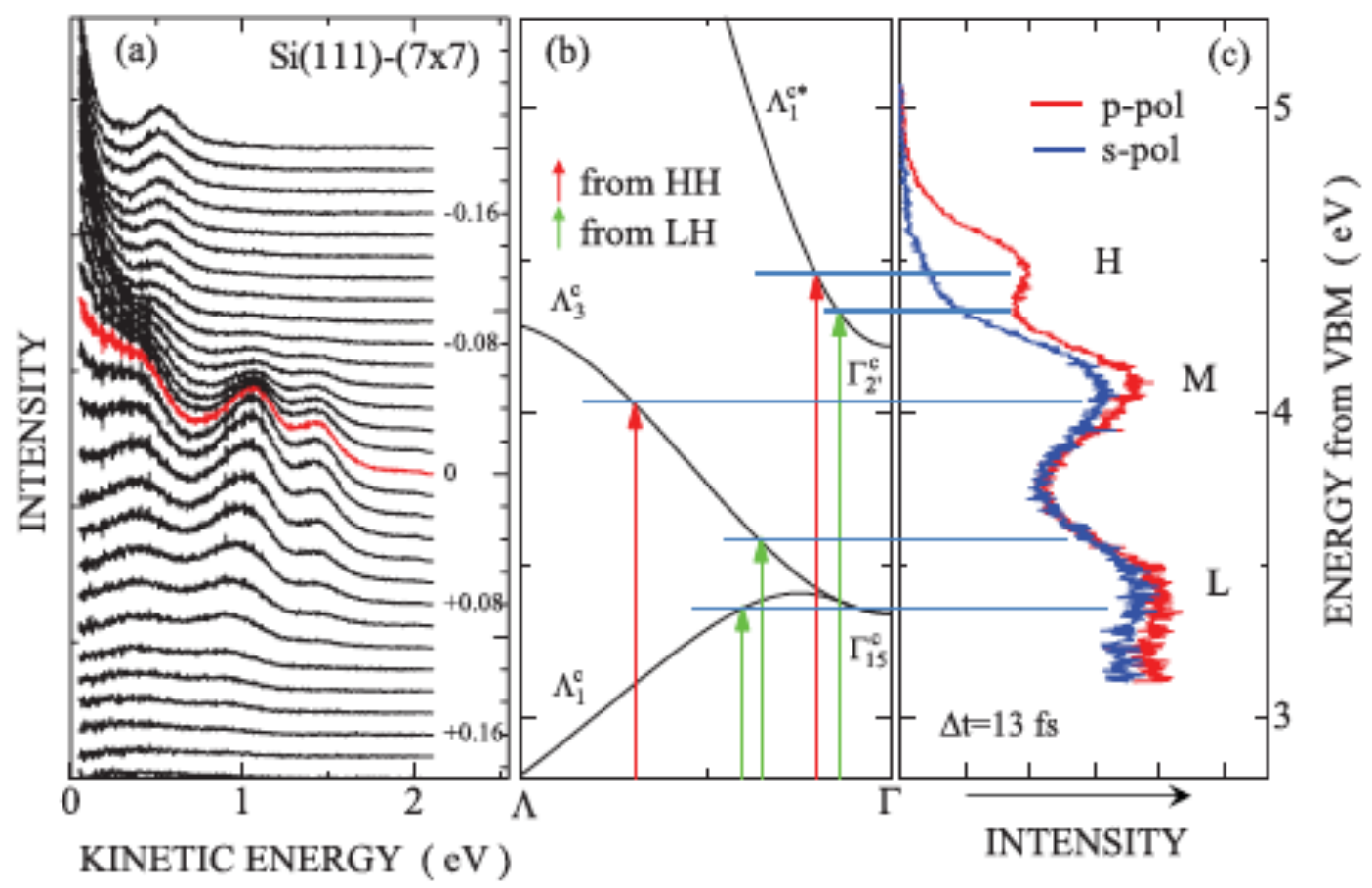

Fig.2 (a) Time-resolved photoemission spectra from $\operatorname{Si}(111)-(7 \times 7)$ obtained using light pulses with two different photon energies: $\mathrm{h} v_{1}=2.21 \mathrm{eV}$ and $\mathrm{h} v_{2}=4.51 \mathrm{eV}$. Both are p-polarized. The time delay, given by numbers in the figure (in ps), defined as the delay of $h v_{1}$ pulses with respect to $h v_{2}$ pulses. The red curve shows the spectrum at $\Delta \mathrm{t}=0$. (b) A part of the conduction-band structure of Si along the $\Gamma-\Lambda$ direction for the energy range from 2.8 to $3.5 \mathrm{eV}$ with respect to the VBM. The red and green arrows show predicted optical transition by p-polarized $4.51-\mathrm{eV}$ photons from the heavy-hole $(\mathrm{HH})$ and light-hole (LH) valence band. (c) Photoemission spectra from Si(111)-(7x7) probed by s- and ppolarized $2.21-\mathrm{eV}$ light pulses $13 \mathrm{fs}$ after excitation with p-polarized $4.51-\mathrm{eV}$ light. The energy is referenced to the VBM. Horizontal lines in (b) and (c) show correspondence from the intermediate states on the band structure in 2PPE processes and observed photoemission peaks. 


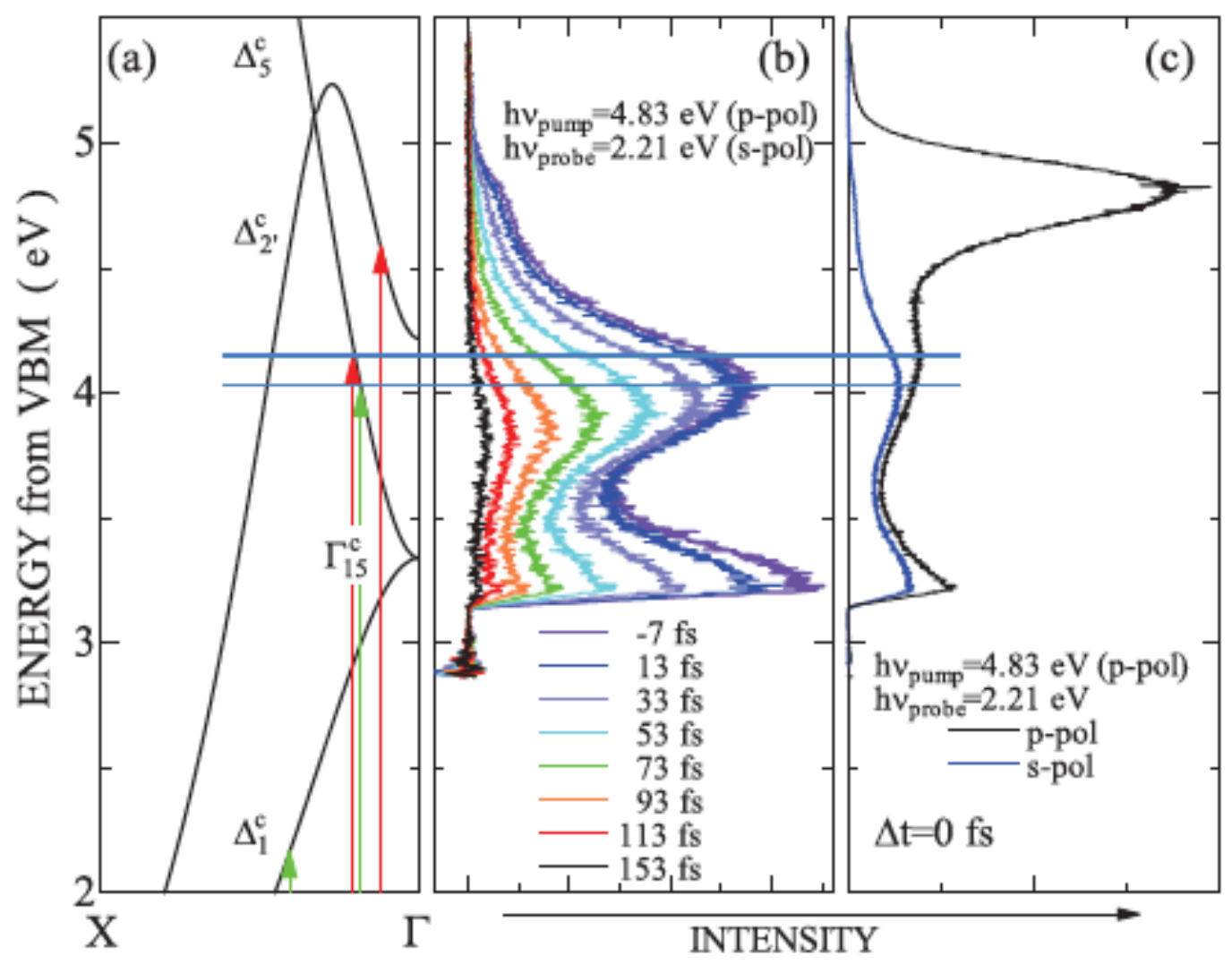

Fig.3 (a) A part of the conduction-band structure of Si along the $\Gamma$-X direction for the energy range from 2.0 to $5.5 \mathrm{eV}$ with respect to the VBM. The red and green arrows show predicted optical transition by p-polarized 4.83-eV photons from the heavy-hole and light-hole valence band. (b) Time-resolved photoemission spectra from $\operatorname{Si}(001)-(2 \times 1)$ obtained using light pulses with two different photon energies: $\mathrm{h} v_{1}=2.21 \mathrm{eV}$ and $\mathrm{h} v_{2}=4.83 \mathrm{eV}$. The $2.21-\mathrm{eV}$ light is s-polarized, while $4.83-\mathrm{eV}$ light is $\mathrm{p}$ polarized. The time delay, given by numbers in the figure, is defined as the delay of $h v_{1}$ pulses with respect to $h v_{2}$ pulses. (c) Time-resolved photoemission spectra from $\operatorname{Si}(001)-(2 \times 1)$ probed by spolarized (blue) and p-polarized (black) 2.21-eV light at $\Delta \mathrm{t}=13 \mathrm{fs}$ after excitation with p-polarized 4.83-eV light. In (b) and (c), the kinetic energies of photoelectrons are referenced to the VBM. Horizontal lines in (b) and (c) show correspondence from the intermediate states on the band structure in 2PPE processes and observed photoemission peaks. 


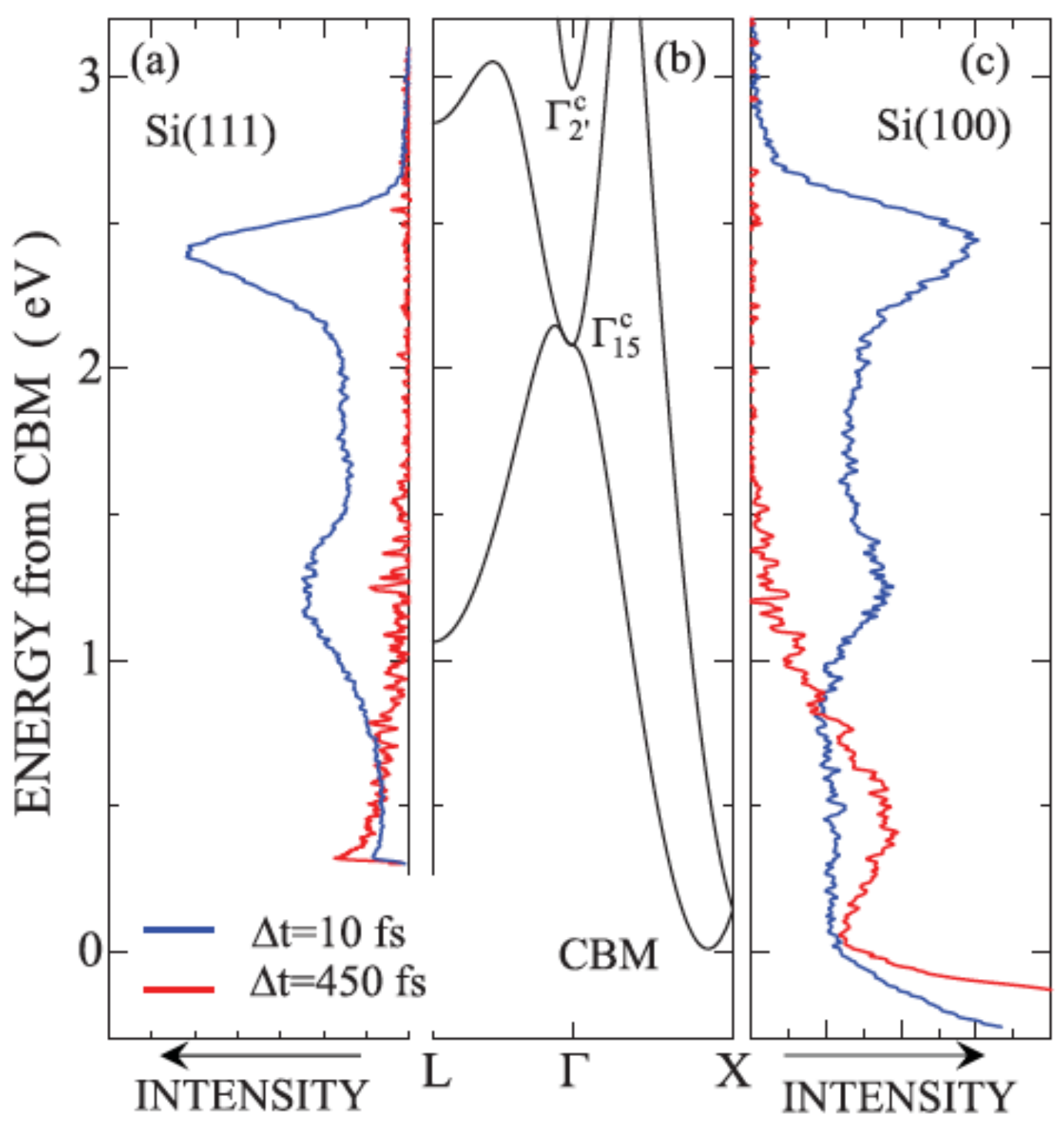

Fig.4 (a) Normal photoemission spectra probed by p-polarized $4.67-\mathrm{eV}$ light at $\Delta \mathrm{t}=10 \mathrm{fs}$, blue, and at $450 \mathrm{fs}$, red, for $\mathrm{Si}(111)-(7 \times 7)$ excited with s-polarized 3.59-eV light. (b) Conduction-band structure of Si along the L- $\Gamma-X$ directions. Energy is referenced with the CBM, thus the scale is that of $\mathrm{E}_{\mathrm{ex}}$. (c) Normal photoemission spectra probed by p-polarized $4.67-\mathrm{eV}$ light at $\Delta \mathrm{t}=10 \mathrm{fs}$, blue, and at $450 \mathrm{fs}$, red, for $\operatorname{Si}(001)-(2 \times 1)$ excited with s-polarized 3.59-eV light. In (a) and (c), the kinetic energies of photoelectrons are referenced to the CBM, based on the spectral line-shape analysis of the photoemission peak from the CBM. 


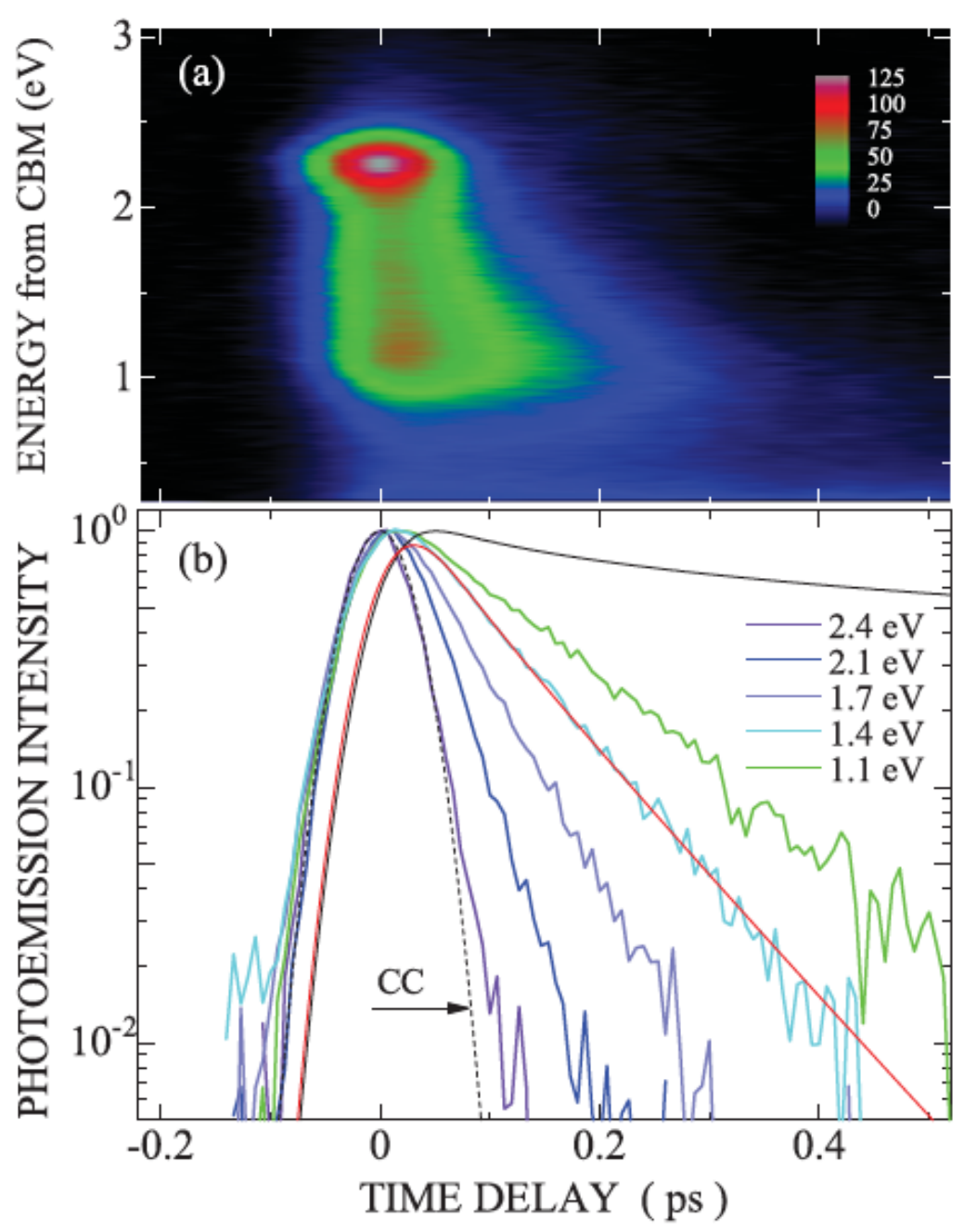

Fig.5 (a) Photoemission image and dynamics of hot electrons injected into the $\Gamma$-L direction by 3.59eV light pulses for $\mathrm{Si}(111)$. Normal photoemission intensities are plotted as a function of energy and time delay. The color scale indicates the photoemission intensity with a linear scale. (b) Temporal changes in the photoemission intensities from hot electrons injected in the $\mathrm{E}_{\mathrm{ex}}$ range from 1.1 to 2.4 eV plotted on a semi-logarithmic scale. The broken curve, labelled CC, shows a Gaussian curve, centered at $\Delta \mathrm{t}=0$, with the width (full width at half maximum) of $65 \mathrm{fs}$, which represents the crosscorrelation trace of pump and probe pulses. As the overlap between pump and probe pulses can be neglected at $\Delta \mathrm{t}>100 \mathrm{fs}$, decay times can be determined almost uniquely from the plot. The solid black and red curves show results of analysis using diffusion-equation model (see the text). 


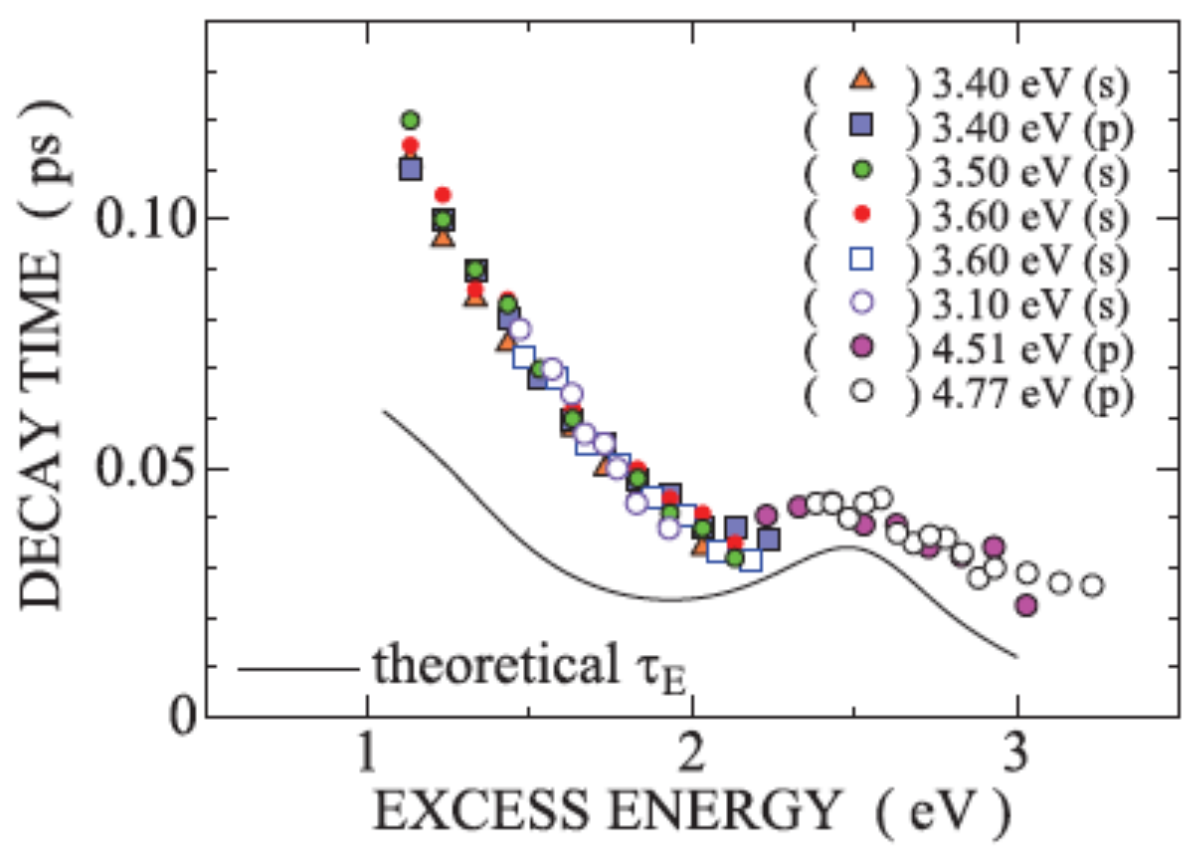

Fig.6 Time constant of population decay of hot electrons as a function of the excess energy with respect to the CBM. Solid symbols represent results obtained for $\mathrm{Si}(111)-(7 \times 7)$, while open symbols show the results for $\mathrm{Si}(001)-(2 \mathrm{x} 1)$. Different symbol styles correspond to different pump-photon energies and polarizations as indicated in the figure. The solid black curve shows the theoretical results of energyrelaxation time due to the electron-phonon scattering for the energy loss interval of $140 \mathrm{meV}$ (see the text). 


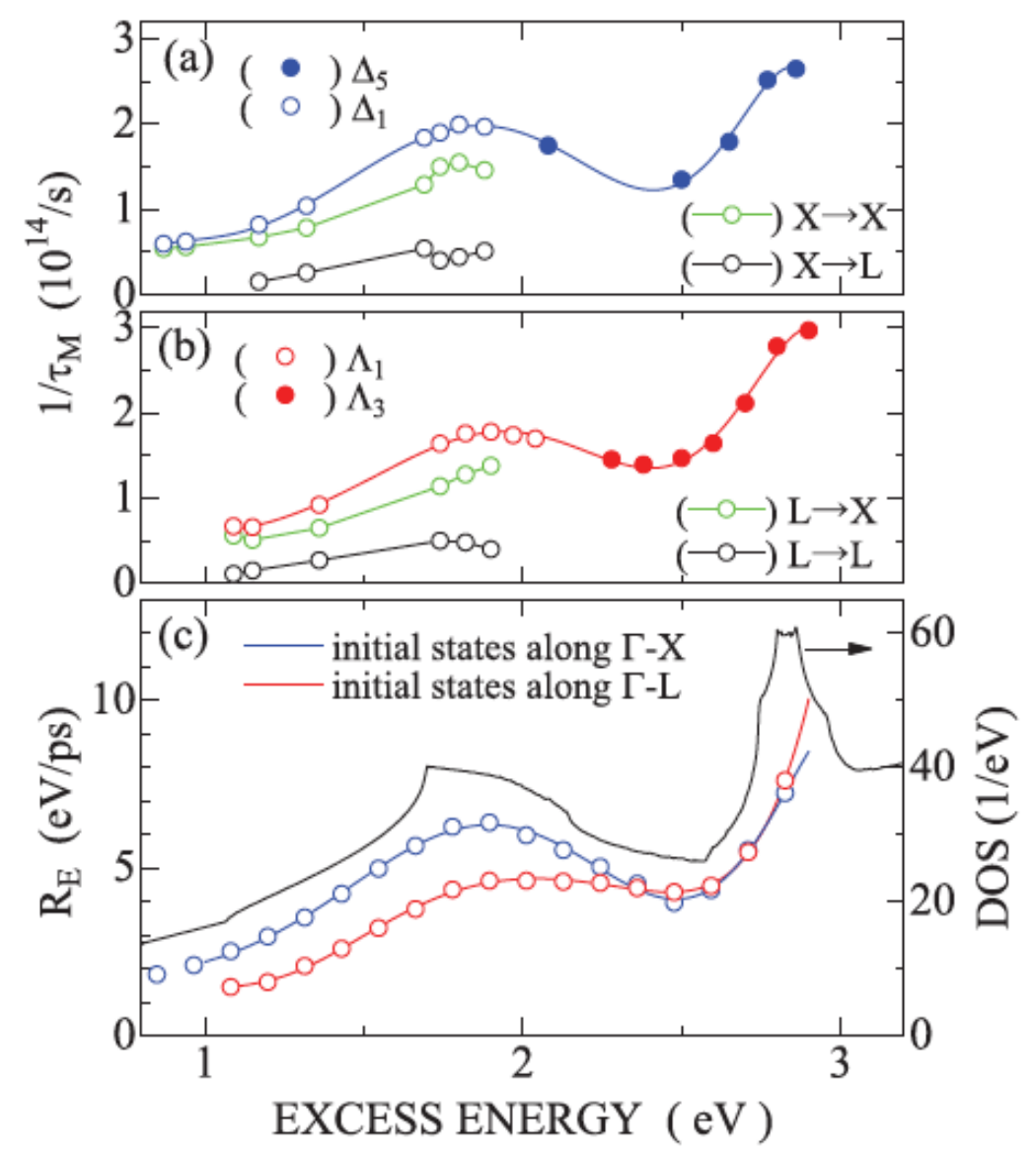

Fig.7 Theoretical results on electron-phonon interactions leading to momentum and energy relaxations.

(a) The momentum scattering rates (in unit of $\Gamma_{M}^{n, k} / \hbar$ ) of hot electrons excited initially in the conduction-band states along the $\Gamma$ to $\mathrm{X}$ direction, as a function of excess energy from the CBM. Not only the total scattering rates but the rates of scattering from a given state to the states in specific valley regions are plotted for $\mathrm{E}_{\mathrm{ex}}<1.9 \mathrm{eV}$ with different symbols indicated in the figure. (b) The momentum scattering rates of hot electrons (in unit of $\Gamma_{M}^{n, k} / \hbar$ ) for the electrons excited in the conduction-band states along the $\Gamma$ to $\mathrm{L}$ direction, as a function of excess energy from the CBM. Not only the total scattering rates but the rates of scattering from a given state to the states in specific valley regions are plotted for $\mathrm{E}_{\mathrm{ex}}<1.9 \mathrm{eV}$ with different symbols indicated in the figure. (c): The energy relaxation rate $\mathrm{R}_{\mathrm{E}}^{\mathrm{n}, \mathrm{k}}$ evaluated for states excited in the $\Gamma$-X direction, blue, and $\Gamma$ - $\mathrm{L}$ direction, red, as a function of excess energy from the CBM. The solid black curve shows the density of electronic states in the CB of Si calculated in the present study (right-axis scale). 
